\title{
Star-QAM Signaling Constellations for Spatial Modulation
}

\author{
Ping Yang, Yue Xiao, Bo Zhang, Shaoqian Li, Senior Member, IEEE, \\ Mohammed El-Hajjar, Member, IEEE, and Lajos Hanzo, Fellow, IEEE
}

\begin{abstract}
5 Abstract-The performance of spatial modulation (SM)-assisted 6 multiple-input-multiple-output (MIMO) communication systems 7 is highly dependent on the specific amplitude/phase modulation 8 (APM) signal constellation adopted. In this paper, we conceive 9 new star-quadrature amplitude modulation (star-QAM)-aided SM 10 schemes. Our goal is to minimize the system's average bit error 11 probability (ABEP). More specifically, a new class of star-QAM 12 constellations is introduced for $\mathbf{S M}$, which is capable of flexi13 bly adapting ring ratios of the amplitude levels. Then, under a 14 specific MIMO configuration and a predetermined transmission 15 rate, a simple and efficient ring-ratio optimization algorithm is 16 proposed to minimize the ABEP. Moreover, to improve further 17 the performance of our star-QAM-aided SM scheme, a diagonal 18 precoding technique is proposed, and a low-complexity minimum19 distance-based approach is conceived for extracting the precod20 ing parameters. Our numerical results show that the proposed 21 star-QAM-aided SM arrangement provides beneficial system per22 formance improvements compared with the identical-throughput 23 maximum-minimum distance (MMD) QAM and phase-shift key24 ing (PSK) benchmarkers. Moreover, our precoding scheme is 25 capable of further improving the attainable system performance 26 at a modest feedback requirement.
\end{abstract}

27 Index Terms-Constellation optimization, multiple-input28 multiple-output (MIMO), spatial modulation (SM), star29 quadrature amplitude modulation (star-QAM).

\section{INTRODUCTION}

31 PATIAL MODULATION (SM), which maps the informa32 tion bits to two information-carrying entities, namely the an33 tenna indexes and the combined amplitude/phase modulation 34 (APM) constellation, constitutes a promising low-complexity 35 multiple-input-multiple-output (MIMO) transmission tech36 nique [1]-[8]. In a conventional single-input-single-output 37 (SISO) system, the Gray-coded maximum-minimum distance

Manuscript received July 27, 2013; revised December 26, 2013; accepted February 8, 2014. This work was supported in part by the European Research Council under an Advanced Fellow Grant, by the National Science Foundation of China under Grant 61101101, by the Foundation Project of the National Key Laboratory of Science and Technology on Communications under Grant 9140C020404120C0201, and by the Key Laboratory of Universal Wireless Communications, Beijing University of Posts and Telecommunications, Chinese Ministry of Education, under Grant KFKT-2012102. The review of this paper was coordinated by Dr. G. Bauch.

P. Yang and Y. Xiao are with the National Key Laboratory of Science and Technology on Communications, University of Electronic Science and Technology of China, Chengdu 611731, China (e-mail: yplxw@163.com).

B. Zhang, S. Li, M. El-Hajjar, and L. Hanzo are with the School of Electronics and Computer Science, University of Southampton, Southampton SO17 1BJ, U.K. (e-mail: bz2g10@ecs.soton.ac.uk; 1sq@uestc.edu.cn; meh@ ecs.soton.ac.uk; 1h@ecs.soton.ac.uk).

Color versions of one or more of the figures in this paper are available online at http://ieeexplore.ieee.org.

Digital Object Identifier 10.1109/TVT.2014.2306986
(MMD) quadrature amplitude modulation (QAM) constellation 38 minimizes the bit error rate (BER) [9], [10]. However, the 39 advantage of MMD-QAM may be eroded in SM-MIMO sys- 40 tems [11]. This is due to the fact that the BER performance of 41 SM-MIMO systems is jointly determined by the spatial signal 42 (i.e., antenna indexes), by the classic APM constellation, and 43 by their interaction [11]-[18].

Recently, the effects of APM schemes on the performance 45 of SM have been investigated in [11], [14], and [18]. More 46 specifically, in [11], the performance of SM systems relying 47 both on conventional QAM and PSK modulation was studied, 48 demonstrating that, in some MIMO setups, the PSK-modulated 49 SM scheme may outperform the identical-throughput MMD- 50 QAM-aided SM scheme. In [18], the dispersion matrices and 51 the signal constellations were jointly optimized for a near- 52 capacity irregular precoded space-time shift keying (STSK) 53 system, which includes SM as a special case and strikes a 54 flexible rate-diversity tradeoff. It was also shown in [14] that 55 the star-QAM-aided STSK scheme outperforms its MMD- 56 based square-QAM-aided counterpart. This observation may 57 be also valid for SM systems [11]. The aforementioned results 58 indicated that the performance of SM is highly dependent on 59 the specific APM adopted; hence, a suitable APM scheme has 60 to be designed for this hybrid modulation scheme.

61

On the other hand, star-QAM constitutes a special case of 62 circular amplitude- and phase-shift keying, which is capable of 63 outperforming the classic square-QAM constellation in peak- 64 power-limited systems [19]. Hence, it has been adopted in most 65 of the recent satellite communication standards, such as in 66 the Digital Video Broadcast System (DVB) S2, DVB-SH, and 67 the Internet Protocol over Satellite and Advanced Broadcasting 68 System via Satellite [19]. The star-QAM constellation is com- 69 posed of multiple concentric circles, and it was shown to be 70 beneficial in the context of STSK systems. Hence, star-QAM 71 may be an attractive APM candidate for SM-MIMO. However, 72 the constellations' optimization has not been carried out for 73 star-QAM-aided SM.

Moreover, to increase the robustness of the SM-MIMO sys- 75 tem, limited-feedback-aided link adaptation schemes have been 76 proposed in [20]-[26]. For example, in [20], an opportunistic 77 power-allocation (PA) scheme was conceived for achieving a 78 beneficial transmit diversity gain in SM-MIMO systems. In 79 [21], a beamforming codebook was designed for optimizing the 80 coding gain of SM-MIMO based on the knowledge of the chan- 81 nel envelope's spatial correlation. Recently, an adaptive closed- 82 loop-aided method was invoked for providing both diversity 83 and coding gains in the context of space-shift keying (SSK)[22], 84 
85 which is a special case of SM. However, the scheme proposed 86 for SSK may not be directly applicable to the conventional 87 SM scheme. Moreover, ASM-MIMO architectures relying on 88 different combinations of modulation schemes were proposed 89 in [24], which aimed for maximizing the channel capacity at a 90 predefined target BER, rather than for minimizing the BER. In 91 contrast, in [25] and [26], a transmit precoding (TPC) technique 92 was used for improving the modulated signal design for SM. 93 However, this technique may only be suitable for a new class of 94 SM relying on a single-receiver antenna. For the conventional $95 \mathrm{SM}$, we proposed a near-instantaneously adaptive-modulation96 aided scheme for minimizing the BER [7], which was termed 97 adaptive SM (ASM). Then, we further generalized this paper in 98 [12] and [15], where the implementation complexity of ASM 99 was considerably reduced. However, ASM typically transmits 100 a different number of bits in the different-quality time slots, 101 which may be inconvenient in fixed-rate applications and po102 tentially leads to error propagation in the case of ASM-mode 103 signaling errors.

104 Against this background, the novel contributions of this 105 paper are threefold.

136 The remainder of this paper is organized as follows. In 137 Section II, we conceive a signaling constellation optimization 138 method for star-QAM-aided SM and elaborate both on the 139 choice of our optimization criterion and on the corresponding 140 optimization algorithm. In Section III, we propose a new TPC 141 scheme for enhancing the performance of the star-QAM-aided
SM. Our numerical analysis is carried out in Section IV. Finally, 142 our conclusions are presented in Section V.

\section{Signaling Constellation Optimization}

\section{A. Performance Metric and Star-QAM Constellation}

Consider a flat-fading MIMO channel associated with $N_{t} 146$ transmit antennas (TAs) and $N_{r}$ receive antennas. The $\left(N_{t} \times 147\right.$ 1)-element transmit symbol vector $\mathbf{x}$ is assumed to satisfy 148 $E\left[\mathbf{x x}^{H}\right]=\mathbf{I}_{N_{t}}$, where $\mathbf{I}_{N_{t}}$ denotes an $\left(N_{t} \times N_{t}\right)$-element 149 identity matrix. Then, the transmitted SM symbol $\mathbf{x} \in \mathbb{C}^{N_{t} \times 1} 150$ is given as $\mathbf{x}=s_{l}^{n} \mathbf{e}_{n}$ [21], where $s_{l}^{n}$ is the complex-valued 151 symbol of the APM scheme employed at the $n$th TA. For 152 example, $L$-PSK/QAM is associated with $m_{\mathrm{APM}}=\log _{2}(L) 153$ input bits, whereas $\mathbf{e}_{n}\left(1 \leq n \leq N_{t}\right)$ is selected from the $N_{t^{-}} 154$ dimensional standard basis vectors (i.e., $\mathbf{e}_{1}=[1,0, \ldots, 0]^{T}$ ), 155 according to $\log _{2}\left(N_{t}\right)$ input bits. The corresponding received 156 signal is given by

$$
\mathbf{y}=\mathbf{H x}+\mathbf{n}=\mathbf{h}_{n} s_{l}^{n}+\mathbf{n}
$$

where $\mathbf{H}$ is an $\left(N_{r} \times N_{t}\right)$-element channel matrix, $\mathbf{h}_{n}$ is the 158 $n$th column of $\mathbf{H}$, and the elements of the $N_{r}$-dimensional noise 159 vector $\mathbf{n}$ are Gaussian random variables obeying $\mathcal{C N}\left(0, N_{0}\right) . \quad 160$

In [11], an improved union bound partitions the ABEP 161 expression of SM-MIMO systems into three terms: the $P_{\text {spatial }} 162$ term related to the TA index, the $P_{\text {signal }}$ term related to the APM 163 signals, and the joint term $P_{\text {joint }}$, which depends on both the TA 164 index and on the APM signals. This bound is formulated as

$$
P_{\mathrm{SM}}(\rho) \leq P_{\text {spatial }}(\rho)+P_{\text {signal }}(\rho)+P_{\text {joint }}(\rho) .
$$

This improved union bound is more accurate than the 166 conventional union-bound-based methods, hence facilitating a 167 deeper understanding of the joint impact of spatial and APM 168 signals, as illustrated in [11]. We focus our attention on the sys- 169 tem's performance for transmission over i.i.d. Rayleigh fading 170 channels, which may be readily extended to the Nakagami-m 171 fading model of [11]. Let us assume that $\rho$ is the average SNR, 172 whereas $x_{l}$ and $x_{\hat{l}}$ represent two different APM constellation 173 points, with their modulus values being given as $\beta_{l}$ and $\beta_{\hat{l}}, 174$ respectively. Then, we have

$$
\begin{aligned}
& P_{\text {signal }}(\rho)=\frac{\log _{2}(L)}{\log _{2}\left(N_{t} \cdot L\right)} P_{\mathrm{APM}}(\rho) \\
& P_{\text {spatial }}(\rho)=\frac{\log _{2}\left(N_{t}\right) N_{t}}{2 L \log _{2}\left(N_{t} \cdot L\right)} \sum_{l=1}^{L} \mathcal{F}\left(\rho \beta_{l}^{2}\right) \\
& P_{\text {joint }}(\rho)=A \sum_{l=1}^{L} \sum_{\hat{l} \neq l=1}^{L}\left[B+C D_{H}\left(x_{l} \rightarrow x_{\hat{l}}\right)\right) \\
& \quad \times \mathcal{F}\left(\frac{\rho}{2}\left(\beta_{l}^{2}+\beta_{\hat{l}}^{2}\right)\right] .
\end{aligned}
$$

Here, $P_{\mathrm{APM}}(\rho)$ represents the error probability of conventional 176 $L$-APM, which depends on the ED of the constellation points 177 of APM, whereas $D_{H}\left(x_{l} \rightarrow x_{\hat{l}}\right)$ is the Hamming distance 178 between signals $x_{l}$ and $x_{\hat{l}}$. Here, $A=1 / L \log \left(N_{t} \cdot L\right), B=179$ $N_{t} \log \left(N_{t}\right) / 2$, and $C=\left(N_{t}-1\right)$ are constants for a fixed 180 


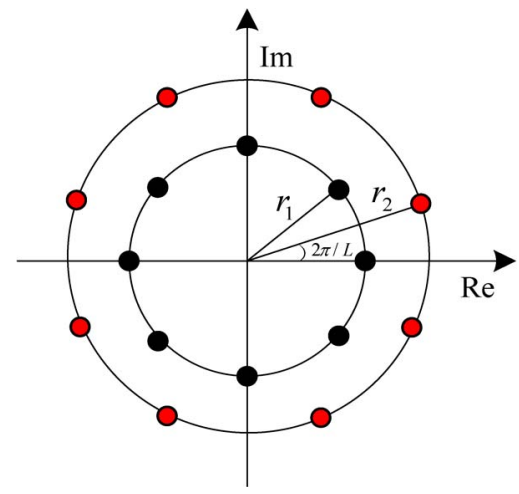

Fig. 1. Complex signal constellation of 16-star-QAM. The symbols are evenly distributed on two rings and the phase differences between the neighboring symbols on the same ring are equal.

181 MIMO setup. Moreover, the function $\mathcal{F}(\varepsilon)$ in (4) and (5) is the 182 pairwise error probability function [11], which is given by

$$
\mathcal{F}(\varepsilon)=\gamma(\varepsilon)^{N_{r}} \sum_{n=0}^{N_{r}-1}\left(\begin{array}{c}
N_{r}-1+n \\
n
\end{array}\right)[1-\gamma(\varepsilon)]^{n}
$$

183 where we have $\gamma(\varepsilon) \triangleq(1 / 2)(1-\sqrt{(\varepsilon / 2+\varepsilon)})$. Note that the 184 ABEP bound of (2) was proposed for the general family of 185 APM schemes, which contains not only the conventional PSK 186 but also the generic rectangular nonsquare-QAM schemes and 187 the square-QAM schemes. Moreover, since $P_{\text {signal }}$ is available 188 in closed form for conventional APM modulation schemes, the 189 bound of (2) is more accurate than the conventional results 190 of [21].

191 As indicated in (3)-(5), $P_{\text {signal }}$ mainly depends on the mini192 mum ED $d_{\min }$ of the APM constellation points, whereas $P_{\text {joint }}$ 193 and $P_{\text {spatial }}$ mainly depend on the modulus values $\beta_{l}(l=$ $1941, \ldots, L)$ of the APM constellation points.

195 Note that the modulus values $\beta_{l}$ are represented by the 196 Frobenius norms of the APM constellation points. These re197 sults suggested that, for jointly minimizing $P_{\text {signal }}, P_{\text {joint }}$, and $198 P_{\text {spatial }}$, we can focus our attention on the design of $d_{\min }$ and 199 on the $\beta_{l}$ parameters of APM.

200 To make the choice of the APM parameters $d_{\min }$ and $\beta_{l}$ as 201 flexible as possible, we consider a class of star-QAM constel202 lations, which subsumes the classic PSK as a special case but 203 may also be configured for maximizing the minimum ED of 204 the constellation by appropriately adjusting the ring ratios of 205 the amplitude levels. For simplicity, we consider the example 206 of a twin-ring 16-star-QAM constellation having a ring ratio 207 of $\alpha=r_{2} / r_{1}$, as shown in Fig. 1. The symbols are evenly 208 distributed on the two rings, and the phase differences between 209 the neighboring symbols on the same ring are equal. Unlike the 210 conventional twin-ring star-QAM constellation [19], [28], the 211 constellation points on the outer circle of our proposed star212 QAM constellation are rotated by $2 \pi / L$ degrees compared 213 with the corresponding constellation points on the inner circle 214 [27]. Hence, again, the conventional PSK constitutes an integral 215 part of our star-QAM scheme, which is associated with $\alpha=1$. 216 Table I summarizes the minimum EDs $d_{\min }$ between the con217 stellation points for different APM schemes. It is found that 218 this star-QAM scheme is capable of achieving almost the same
TABLE I

Minimum ED Between the Constellation POINTS FOR DIFFERENT APM SCHEMES

\begin{tabular}{|l|c|c|c|c|c|}
\hline Modulation order & 2 & 4 (MMD) & $8([9])$ & $16(\mathrm{MMD})$ & $32([9])$ \\
\hline PSK & 2 & $\sqrt{2}$ & 0.7654 & 0.3902 & 0.1960 \\
\hline QAM & -- & $\sqrt{2}$ & 0.8165 & 0.6325 & 0.4082 \\
\hline Proposed star-QAM & 2 & $\sqrt{2}$ & 0.9134 & 0.5737 & 0.3952 \\
\hline
\end{tabular}

minimum ED as the MMD-based QAM. Note that, although 219 this twin-ring star-QAM constellation has been indeed applied 220 for noncoherent detection [27], it has not been considered 221 whether this constellation can be directly applied to SM for 222 achieving performance improvements.

The aforementioned twin-ring philosophy of Fig. 1 may be 224 readily extended to multiple-ring star-QAM. The reasons for 225 considering twin-ring star-QAM in our paper are the following. 226

- It is an attractive APM modulation candidate for SM, 227 exhibiting a high performance at low detection complexity 228 compared with conventional QAM schemes, as detailed in 229 [13]-[15].

- It can be flexibly designed for different $d_{\min }$ and $\beta_{l}(l=231$ $1, \ldots, L)$ combinations, which is achieved by simply ad- 232 justing a single parameter $\alpha$, whereas $\beta_{l}$ can assume two 233 values because only two rings are considered.

- The ABEP of star-QAM, which is related to the $P_{\text {spatial }} 235$ term of (3), has been documented in [28] and [29].

\section{B. Optimization Criteria and Optimization Algorithm}

Observe in Fig. 1 that there are numerous options for the 238 parameter $\alpha$ of the star-QAM constellation, for a given MIMO 239 setup, specified by the total number of bits per symbol $m_{\text {all }}, 240$ the $\left(N_{r} \times N_{t}\right)$ configuration of transceiver, and the number of 241 modulation level $L$. The goal of star-QAM-aided signaling $242 \mathbf{A Q 2}$ constellation optimization is to find the specific ring ratio $\alpha, 243$ which minimizes the ABEP of the SM-MIMO of (2). Note that, 244 although the term $P_{\mathrm{SM}}(\rho)$ in (2) cannot be directly represented 245 by parameter $\alpha$, it varies as a function of $\alpha$, which may 246 be formulated as $P_{\mathrm{SM}}(\rho, \alpha)$. Following the aforementioned 247 approach, we formulated this optimization problem as

$$
\left\{\begin{array}{l}
\alpha^{*}=\min _{\alpha} P_{\mathrm{SM}}(\rho, \alpha) \\
\text { s.t. } \quad \alpha \geq 1
\end{array}\right.
$$

which may be a convex one for a fixed SNR value $\rho$, as 249 indicated in Fig. 4. However, deriving the closed-form solu- 250 tion of (7) remains an open challenge since the expression of 251 $P_{\mathrm{SM}}(\rho, \alpha)$ depends both on the specific APM constellation and 252 on the particular MIMO setup [19], and since the expressions 253 of $P_{\text {signal }}, P_{\text {joint }}$ and $P_{\text {spatial }}$ in (3)-(5) are complex. Hence, a 254 numerical search is adopted.

Our optimization algorithm conceived for finding the ring 256 ratio is summarized as follows.

Step 1: Initialize the values of $N_{r}, N_{t}, m_{\text {all }}, L$, and the SNR 258 value $\rho$. Set the iteration step size to $\Delta \alpha=0.1$ and the 259 number of iterations to $n=1$. The choice of $\Delta \alpha$ is flexible, 260 and a lower value of $\Delta \alpha$ may lead to a better performance. 261 We then set the search area of $\alpha$ to $1 \leq \alpha \leq U_{\alpha}$ and the 262 performance metric to $P_{\text {iter }}(n)=0$. 


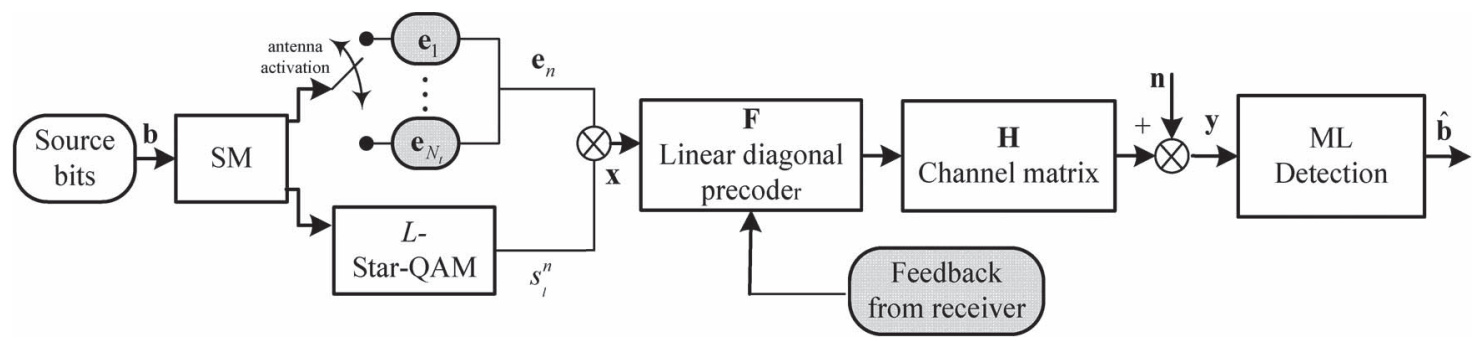

Fig. 2. System model of the diagonal-precoding-assisted star-QAM-aided SM scheme.

264 Step 2: While $\alpha \leq U_{\alpha}$, let $\Delta \hat{\alpha}=\min \left\{\Delta \hat{\alpha}, U_{\alpha}-\alpha\right\}$, and cal265 culate the probabilities of $P_{\text {signal }}, P_{\text {joint }}$, and $P_{\text {spatial }}$ by us266 ing (3)-(5) associated with $\alpha$. Then, let $P_{\text {iter }}(n)=P_{\mathrm{SM}}(\rho)$ 267 using (2), and set $\alpha=\alpha+\Delta \hat{\alpha}$ and $n=n+1$.

268 Step 3 : Find the index $n^{*}=\min _{n}\left\{P_{\text {iter }}(n)\right\}$ to achieve the 269 optimal ring ratio of $\alpha^{*}=1+\left(n^{*}-1\right) \Delta \hat{\alpha}$.

270 In the aforementioned optimization algorithm, we have to 271 choose an appropriate $U_{\alpha}$ to promptly find the optimal $\alpha^{*}$. 272 More explicitly, an excessively low value of $U_{\alpha}$ may lead 273 to missing the optimal solution, whereas an excessively high 274 value of $U_{\alpha}$ imposes excessive computational complexity on 275 the optimization process. Hence, we will show in Section III 276 that $U_{\alpha}=3$ is a beneficial choice for promptly approaching 277 the optimal results. Moreover, the optimum ring ratio $\alpha^{*}$ is a 278 function of the SNR. However, we will show that the optimum 279 ratio approaches its asymptotic optimum as the SNR increases.

\section{III. Proposed Diagonal Precoding For \\ 281 Star-Quadrature Amplitude Modulation-Aided 282 SPATIAL MODUALTION}

283 Since the performance of the optimum maximum-likelihood 284 (ML) receiver depends on the FD of the received signal con285 stellation [30], we propose a new TPC based on maximizing 286 the FD for the family of star-QAM-aided SM-MIMO systems, 287 when limited channel state information is available at the 288 transmitter. Since the FD is increased by the TPC algorithm, 289 the proposed scheme is expected to provide a beneficial system 290 performance improvement. To retain all the single-RF-related 291 benefits of SM, we design the TPC matrix $\mathbf{P}$ to be diagonal. 292 The system model of the diagonal-TPC-assisted star-QAM293 aided SM scheme is shown in Fig. 2. To identify the specific 294 TPC parameters, which are capable of maximizing the FD, 295 we propose a low-complexity TPC design algorithm. We will 296 demonstrate that as few as two elements of the diagonal TPC 297 matrix have to be fed back to the transmitter, regardless of $N_{t}$.

\section{A. TPC Design Criterion}

299 To construct a TPC for star-QAM-aided SM-MIMO systems, 300 we can rewrite the system model of (1) as

$$
\mathbf{y}=\mathbf{H P x}+\mathbf{n}
$$

301 where $\mathbf{P}$ denotes the diagonal TPC matrix, which can be 302 represented as

$$
\mathbf{P}=\operatorname{diag}\left\{p_{1}, \ldots, p_{n}, \ldots, p_{N_{t}}\right\}
$$

where $p_{n}$ controls the channel gain associated with $x_{n}$. Here, 303 we let $\sum_{n=1}^{N_{t}}\left|p_{n}\right|^{2}=N_{t}$ for normalizing the transmit power. 304 Note that the introduction of TPC in SM does not affect the 305 advantages of SM, such as the avoidance of the interantenna 306 interference and the reliance on a single RF chain, because the 307 precoded transmit vector $\mathbf{P x}$ includes only a single nonzero 308 component; hence, only a single TA is activated in each time 309 slot, as indicated in (8).

310

Numerous techniques may be invoked for constructing the 311 TPC $\mathbf{P}$ [21], [25]. In this paper, similar to the precoding 312 methods conceived for the orthogonalized spatial multiplexing 313 of [31], we decompose $\mathbf{P}$ as

$$
\mathbf{P}=\overline{\mathbf{P}} \boldsymbol{\Theta}=\operatorname{diag}\left\{\bar{p}_{1} e^{j \theta_{1}}, \ldots, \bar{p}_{n} e^{j \theta_{n}}, \ldots, \bar{p}_{N_{t}} e^{j \theta_{N_{t}}}\right\}
$$

where $\overline{\mathbf{P}}=\operatorname{diag}\left\{\bar{p}_{1}, \ldots, \bar{p}_{n}, \ldots, \bar{p}_{N_{t}}\right\}$ represents the PA ma- 315 trix, whereas $\boldsymbol{\Theta}=\operatorname{diag}\left\{e^{j \theta_{1}}, \ldots, e^{j \theta_{n}}, \ldots, e^{j \theta_{N_{t}}}\right\}$ is the phase 316 rotation matrix. The FD between the constellation points at the 317 receiver is defined as

$$
\begin{aligned}
d_{\min }(\mathbf{H}, \mathbf{P}) & =\min _{\substack{\mathbf{x}_{i}, \mathbf{x}_{j} \in \mathbb{X}, \mathbf{x}_{i} \neq \mathbf{x}_{j}}}\left\|\mathbf{H P}\left(\mathbf{x}_{i}-\mathbf{x}_{j}\right)\right\|_{F} \\
& =\min _{\mathbf{e}_{i j} \in \mathbb{E}}\left\|\mathbf{H} \overline{\mathbf{P}} \boldsymbol{\Theta} \mathbf{e}_{i j}\right\|_{F}
\end{aligned}
$$

where $\mathbb{X}$ is the set of all legitimate transmit symbols, $\mathbf{e}_{i j}=319$ $\mathbf{x}_{i}-\mathbf{x}_{j}, i \neq j$ denotes the error vector, and $\mathbb{E}$ is a set of error 320 vectors. Then, we design the TPC $\mathbf{P}$ by maximizing the FD 321 with the aid of the following criterion:

$$
\left\{\begin{array}{l}
\mathbf{P}_{\mathrm{opt}}=\underset{\mathbf{P}}{\arg \max } d_{\min }(\mathbf{H}, \mathbf{P}) \\
\text { s.t. } \quad \sum_{n=1}^{N_{t}}\left|p_{n}\right|^{2}=N_{t} ; \quad p_{n} \in C ; \\
\\
\theta_{n} \in(0,2 \pi] ; \quad n=1, \ldots, N_{t} .
\end{array}\right.
$$

Note that, since the attainable performance of the optimum 323 single-stream ML receiver depends on the FD of the received 324 signal constellation [30], the maximization of the FD directly 325 reduces the probability of error. ${ }^{1}$ Let $\mathbf{x}_{i}=s_{l}^{i} \mathbf{e}_{i}$ and $\mathbf{x}_{j}=s_{k}^{j} \mathbf{e}_{j} 326$ denote two different transmit symbols, whereas $s_{l}^{i}$ and $s_{k}^{j} 327$ denote the constellation points $l$ and $k$ represented by the $i$ th 328 and $j$ th antennas, respectively. Then, the FD of (11) can be 329 represented as (13), where $\phi=\angle\left(\left(s_{l}^{i}\right)^{*} s_{k}^{j}\right)=-\left(s_{l}^{i}\left(s_{k}^{j}\right)^{*}\right)$. In 330

\footnotetext{
${ }^{1}$ Because the conventional PSK-and-QAM-aided SM scheme's performance is worse than that of the proposed star-QAM-aided SM, we only invoked the TPC algorithm for the star-QAM-aided SM for the sake of achieving further performance improvements. However, it is worth noting that the proposed TPC algorithm is also suitable for SM in conjunction with both conventional PSK and QAM schemes.
} 
331 the ASM scheme of [7], only the APM modulation orders to 332 be used by the transmitter are adapted, i.e., only the elements $333\left|s_{l}^{i}\right|,\left|s_{k}^{j}\right|$, and $\phi$ of (13), shown at the bottom of the page, are 334 dynamically adapted to the channel conditions, and the legit335 imate values of these elements are selected from the discrete 336 set depending on the modulation order set utilized. By contrast, 337 our proposed scheme adjusts all the TPC elements $\left|p_{i}\right|,\left|p_{j}\right|$, $338 \theta_{i}$, and $\theta_{j}$ of (13) for maximizing the FD $d_{\min }(\mathbf{H}, \mathbf{P})$, whose 339 legitimate values are drawn from the real-valued number field. 340 Based on these observations and on (13), the proposed scheme 341 and the ASM scheme may exploit the same degrees of freedom 342 as that offered by the SM-MIMO in terms of maximizing the 343 FD. However, unlike the ASM scheme of [7] and [15], our 344 proposed scheme assigns the same number of bits to each time 345 slot; hence, the potential error propagation effects experienced 346 in ASM are avoided.

\section{B. Low-Complexity TPC Design Algorithm}

348 To identify the specific TPC matrix $\mathbf{P}$, which is capable of 349 maximizing the $\mathrm{FD}$, we have to determine all the $N_{t}$ parameters $350 p_{n}\left(n=1, \ldots, N_{t}\right)$. Since it may become excessively complex 351 to jointly optimize these $N_{t}$ parameters in the complex-valued 352 field, we propose a low-complexity precoder design algorithm. 353 Similar to the one-bit reallocation algorithm designed for ASM 354 in [15], only the specific TA pair associated with the FD is con355 sidered, and the TPC parameters are selected for appropriately 356 weighting the SM symbols because the FD of this particular 357 TA pair predominantly determines the achievable performance. 358 The calculation of the TPC matrix is summarized in Fig. 3.

359 To be specific, given the channel matrix $\mathbf{H}$, the indexes of 360 the TA pair $(g, k)$ associated with the FD $d_{\min }(\mathbf{H})$ can be 361 found with the aid of the flowchart shown in Fig. 3. To offer 362 an increased FD, the precoding parameters of this TA pair can 363 be dynamically adapted. Note that, if the value of $g$ is the same 364 as $k$, it is plausible that the TA $g$ has the smallest channel gain. 365 In this case, the phase rotation elements of (10) do not have to 366 be considered because this would not increase the FD of (13). 367 To increase the FD, we only consider the PA matrix of (10) 368 and may deduct some power from the TA $u$ having the highest 369 channel gain, which may hence be reassigned it to the TA $g$. 370 As a result, $p_{u}$ and $p_{g}$ have to be optimized. On the other hand, 371 if the value of $g$ and $k$ is not the same, parameters $p_{g}$ and $p_{k}$ 372 have to be calculated. Overall, there are only two parameters, 373 namely, $p_{g}$ and $p_{k},\left(p_{u}\right.$ for $\left.g=k\right)$ that have to be searched 374 for. Finding the optimal values of $p_{g}$ and $p_{k}$ as a function of

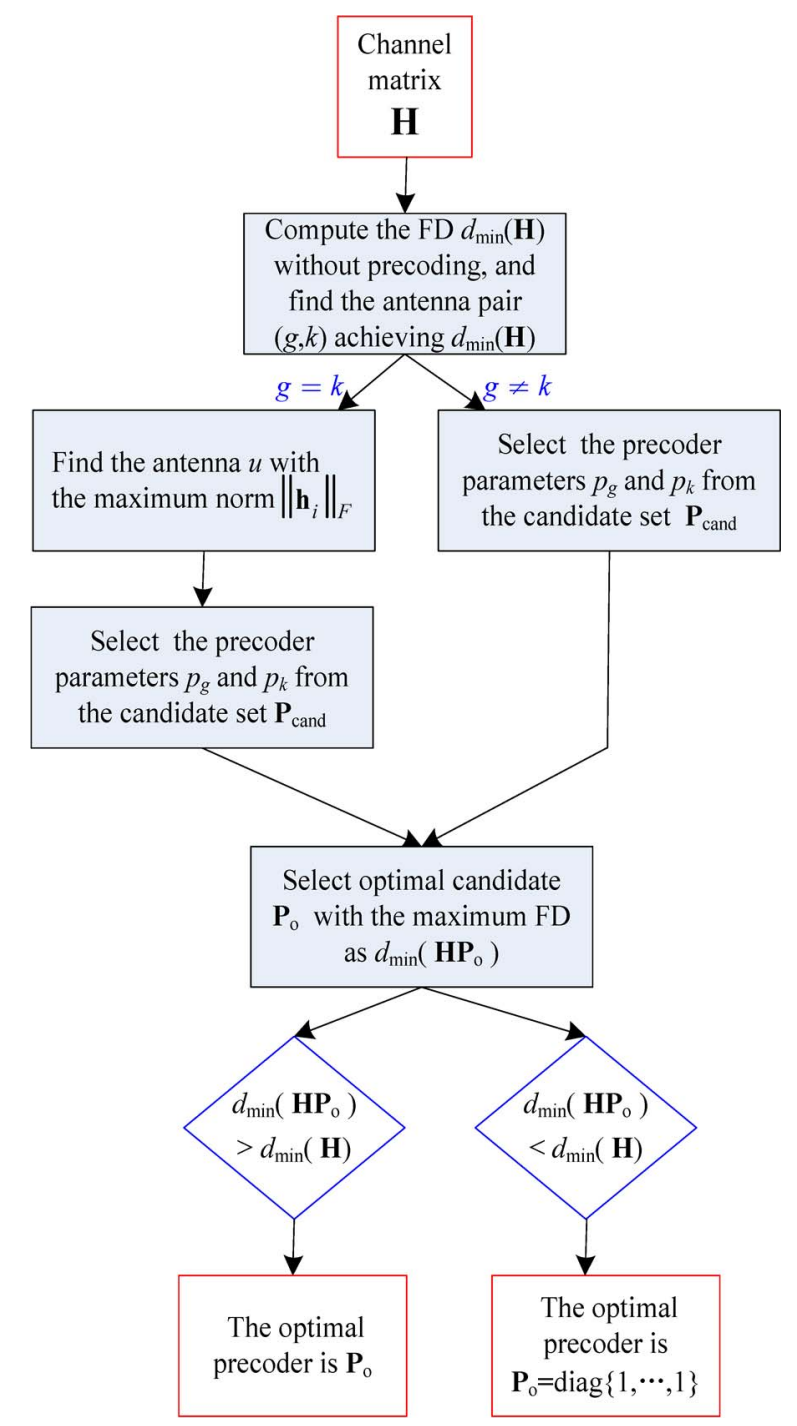

Fig. 3. Calculation of the diagonal precoding matrix for star-QAM-aided SM-MIMO.

both $\mathbf{H}$ and of the optimal transmit parameters involves an 375 exhaustive search over the vast design space of $\bar{p}_{g}, \bar{p}_{k}, \theta_{g}$, and 376 $\theta_{k}$ of (10), which is overly complex. To reduce the complexity, 377 according to (12), the power of the TA pair $(g, k)$ satisfies the 378 constraint $\bar{p}_{k}^{2}+\bar{p}_{g}^{2}=2$; hence, only the element $\bar{p}_{k}$ has to be 379 searched for in the power matrix $\overline{\mathbf{P}}$ of (10). Moreover, since 380 the phase rotation of the symbol is only carried by two TAs 381 and their phase difference is correlated, we can simplify the 382 computations by fixing $\theta_{k}=1$ and then finding the optimal 383

$$
\begin{aligned}
d_{\min }(\mathbf{H}, \mathbf{P}) & =\min _{s_{l}^{j}, s_{k}^{j} \in S}\left\|\mathbf{H P}\left(s_{l}^{i} \mathbf{e}_{i}-s_{k}^{j} \mathbf{e}_{j}\right)\right\|_{F} \\
& =\min _{s_{l}^{j}, s_{k}^{j} \in S}\left\|\left(\mathbf{h}_{i} p_{i} s_{l}^{i}-\mathbf{h}_{j} p_{j} s_{k}^{j}\right)\right\|_{F} \\
& =\min _{s_{l}^{j}, s_{k}^{j} \in S} \sqrt{\left|s_{l}^{i}\right|^{2}\left|p_{i}\right|^{2} \mathbf{h}_{i}^{\mathrm{H}} \mathbf{h}_{i}+\left|s_{k}^{j}\right|^{2}\left|p_{j}\right|^{2} \mathbf{h}_{j}^{\mathrm{H}} \mathbf{h}_{j}-2\left|p_{i}\right|\left|p_{j}\right|\left|s_{l}^{i}\right|\left|s_{k}^{j}\right| \operatorname{Re}\left\{\mathbf{h}_{i}^{\mathrm{H}} \mathbf{h}_{j} e^{j\left(\phi-\theta_{i}+\theta_{j}\right)}\right\}}
\end{aligned}
$$


$384 \theta_{g}$. This implies that only the phase parameter $\theta_{g}$ has to be 385 optimized for the phase matrix $\Theta$. In Fig. 3, a numerical search 386 is used for varying $\bar{p}_{g}$ and $\theta_{g}$ in small steps. Note that we 387 have $0 \leq \bar{p}_{g} \leq \sqrt{2}$ and $0 \leq \theta_{g} \leq 2 \pi$ according to (12). For our 388 numerical search, we have assumed

$$
\begin{cases}\bar{p}_{g}=\sqrt{2} / V_{1} * v_{1}, & v_{1}=0, \ldots, V_{1} \\ \theta_{g}=2 \pi / V_{2} * v_{2}, & v_{2}=0, \ldots, V_{2}\end{cases}
$$

389 where $V_{1}$ and $V_{2}$ represent the number of quantization steps and 390 can be flexibly selected according to the prevalent performance 391 requirements. As a result, the corresponding diagonal TPC 392 matrix candidates are

$$
\begin{gathered}
\mathbf{P}_{\text {cand }}=\operatorname{diag}\left\{1, \ldots, \bar{p}_{g} e^{j \theta_{g}}, \ldots, \sqrt{2-\bar{p}_{g}^{2}}, \ldots, 1\right\} \\
\uparrow g \text { th } \quad \uparrow k \text { th. }
\end{gathered}
$$

393 Upon denoting the quantized TPC matrix $\mathbf{P}$ as $\mathbf{P}_{\text {cand }}$, the 394 optimization problem of (12) is reformulated as

$$
\mathbf{P}_{\mathrm{opt}}=\underset{\left\{\mathbf{P} \in \mathbf{P}_{\text {cand }}, \mathbf{P}_{\mathrm{I}}\right\}}{\arg \max } d_{\min }(\mathbf{H}, \mathbf{P}) .
$$

395 where we have $\mathbf{P}_{\mathrm{I}}=\mathbf{I}_{N_{t}}$. In (16), the FD of the TPC matrices $396 \mathbf{P}_{\text {cand }}$ generated will be compared with that of the conventional 397 scheme associated with $\mathbf{P}_{\mathrm{I}}$, and then we select the one having 398 the largest FD as our final result. The receiver determines the 399 optimal diagonal TPC matrix based on (16) and feeds back the 400 TA indexes and their TPC parameters to the transmitter. Since 401 only the specific TA pair, which predominantly determines 402 the achievable performance, is considered, the proposed low403 complexity algorithm can be readily extended to a high number 404 of TAs.

\section{Simulation Results}

406 Here, we characterize the performance of both the proposed 407 star-QAM-aided SM scheme and of the corresponding TPC 408 scheme, and compare it with that of the conventional QAM409 modulated SM schemes, with the PSK-modulated SM schemes 410 and with the ASM schemes [15] for transmission over inde411 pendent Rayleigh block-flat MIMO channels. It is assumed that 412 the receiver is capable of perfect phase and gain tracking, i.e., 413 of perfect channel estimation. In practice, pilot symbols are 414 used for estimating the MIMO channel; hence, the estimated 415 channel matrix will inevitably be imperfect. To alleviate the 416 effects of channel estimation errors, the joint channel estimation 417 and data detection algorithm of [32] may be considered in the 418 proposed schemes, where the channel estimator and the data 419 detector iteratively exchange their information. We consider 420 two practical MIMO systems here, namely, $(2 \times 2)$ and $(4 \times$ $4214)$ MIMO systems. Moreover, in the TPC design algorithm, we 422 select $V_{1}=V_{2}=5$ for simplicity. ${ }^{2}$

423 Fig. 4 shows the optimal ring ratios of star-QAM-aided 424 SM relying on $(4 \times 4)$ elements for a different number of 425 modulation levels $L$, where the optimal ring ratio $\alpha^{*}$ is seen 426 to be a function of the SNR. The bound of (2) is well suited

\footnotetext{
${ }^{2}$ Note that the values of $V_{1}$ and $V_{2}$ can be different. Moreover, the selection of $V_{1}$ and $V_{2}$ is flexible, and higher values of $V_{1}$ and $V_{2}$ may lead to better performance at the cost of a higher TPC design complexity.
}

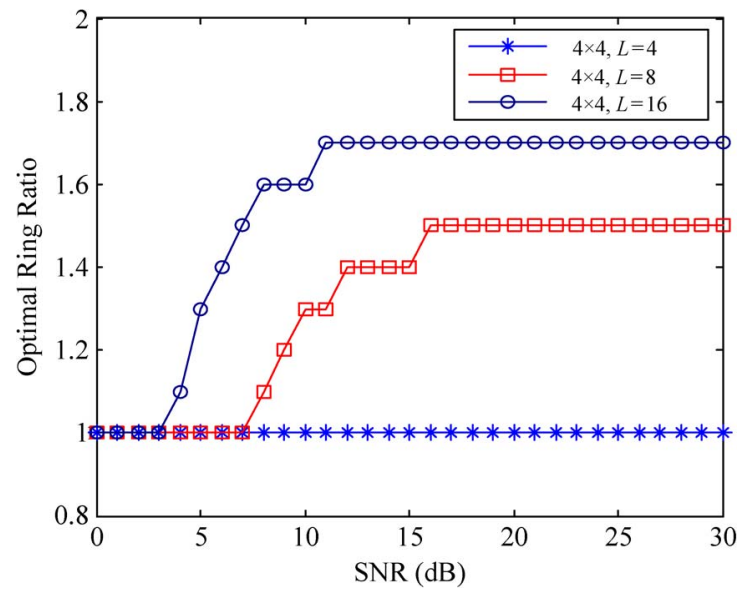

Fig. 4. Optimal ring ratios of star-QAM-aided SM with $(4 \times 4)$ MIMO for different numbers of modulation levels $L$.

for numerically optimizing the ring ratio, particularly in the 427 high-SNR region. Observe in Fig. 4 that the optimal ratios 428 approach their asymptotic values, as the SNR increases. This 429 is expected since the bound of (2) is also asymptotically tight 430 and the probability of an error event in slow fading associated 431 with ML detection is dominated by the minimum-distance error 432 event at high values of the SNR. Moreover, the optimal ring 433 ratios are different for different MIMO parameters.

434

Since the transmitter operates at a fixed ring ratio, we have 435 opted for the asymptotic ring-ratio value for the evaluation 436 of the BER. For example, we have chosen the optimal ring 437 ratio $\alpha^{*}=1.7$ for the 16 -star-QAM-aided $(4 \times 4)$ SM-MIMO, 438 according to the results in Fig. 4. This result may be readily 439 extended to other star-QAM-aided SM scenarios, such as the (4 440 $\times 4$ )-element star-QAM-aided SM schemes using $L=4,8$ in 441 Fig. 4.

In Figs. 5 and 6, we compare various SM-MIMO systems 443 relying on diverse MIMO parameters and modulation orders. 444 First, in Figs. 5 and 6, we depict the BER performance 445 of the conventional QAM-modulated SM schemes, of the 446 PSK-modulated SM arrangements, and of the proposed star- 447 QAM-aided SM scheme. Note that the optimized star-QAM 448 constellation is designed offline for different SM-MIMO sys- 449 tems. Hence, the resultant system does not need any feedback. 450 To be specific, we may create a parameter lookup table for 451 the star-QAM SM schemes associated with the MIMO setups 452 considered; hence, the complexity of the optimal ring-ratio 453 search process detailed in Section II is negligible. For com- 454 pleteness, we also included the theoretical upper bound [30] 455 for the family of conventional SM schemes. We found that 456 the conventional QAM-modulated SM scheme outperforms its 457 identical-throughput PSK counterpart for a $(4 \times 4)$-element 458 MIMO channel in Fig. 5, whereas the PSK scheme is preferred 459 for a $(2 \times 2)$-element MIMO channel in Fig. 6 . This indicates 460 that the best choice of the APM scheme depends on the specific 461 SM parameters, such as the MIMO setup and throughput. 462 Moreover, as shown in Fig. 5, the optimized star-QAM-aided 463 SM scheme provides an SNR gain of about $3 \mathrm{~dB}$ at BER $=464$ $10^{-5}$ over the conventional 16-PSK-modulated SM scheme and 465 an SNR gain of about $1.1 \mathrm{~dB}$ over the identical-throughput 466 


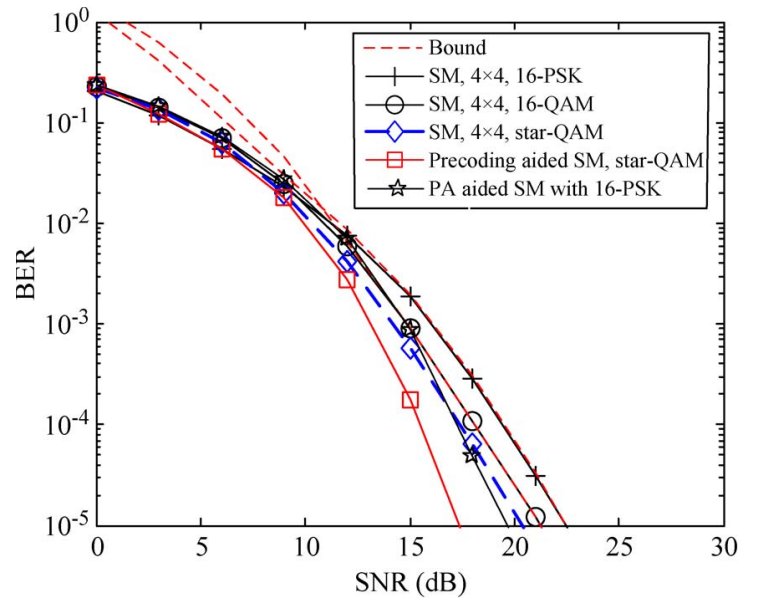

Fig. 5. BER performance of various SM-MIMO schemes operating in a $(4 \times$ 4) MIMO channel at a total throughput of $6 \mathrm{~b} / \mathrm{s}$. Since the transmitter operates with a fixed ring ratio, we have chosen the asymptotic ring-ratio value for the evaluation of star-QAM-aided schemes. Here, $\alpha$ is chosen as $\alpha=1.7$.

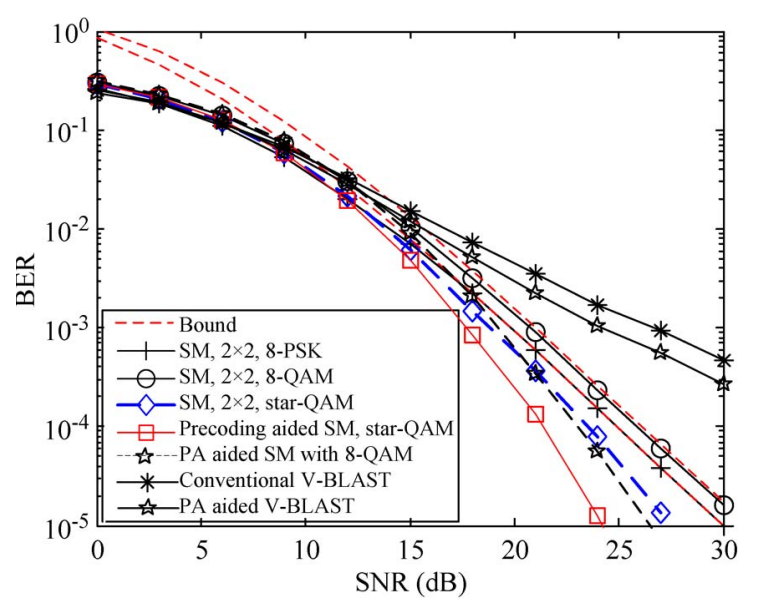

Fig. 6. BER performance of various SM schemes operating in $(2 \times 2)$ MIMO channel at a total throughput of $4 \mathrm{~b} / \mathrm{s}$. Here, $\alpha$ is chosen as $\alpha=1.5$.

467 Gray-coded MMD 16-QAM SM scheme. This advantage of the 468 optimized star-QAM scheme recorded for SM-MIMO is also 469 visible in Fig. 6.

470 Moreover, in Figs. 5 and 6, we also compare the 471 achievable BER performance of the limited-feedback-aided 472 ASM schemes. To be specific, two diagonal-precoding-aided 473 schemes, namely, the precoding-assisted star-QAM-based SM 474 schemes and the PA-aided SM schemes of [33] are compared. 475 For simplicity, the PA algorithm is only applied to the non476 ASM schemes exhibiting an inferior performance in Figs. 5 477 and 6, namely, to the conventional $(4 \times 4)$-element SM using 47816 -PSK and $(2 \times 2)$-element SM employing 8-QAM. Note that 479 the $(4 \times 4)$-element $\mathrm{SM}$ associated with 16 -QAM and $(2 \times$ 480 2)-element SM employing 8-PSK can also use the PA regime 481 to attain a BER improvement. Due to space limitations, these 482 results are not presented here. As shown in Figs. 5 and 6, the 483 proposed TPC schemes provide a gain of $2.5-3 \mathrm{~dB}$ at the BER 484 of $10^{-5}$ over the PA-aided SM schemes. This is because PA485 aided SM may be viewed as a special case of the proposed 486 precoding-aided SM created by only considering the PA matrix 487 in (10). To be specific, compared with the PA-aided SM of

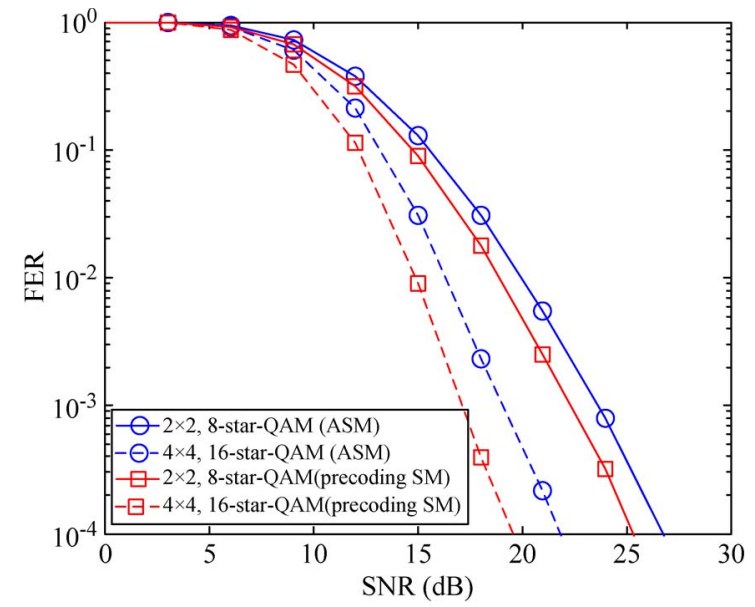

Fig. 7. FER performance of the proposed precoding star-QAM-aided SM and the ASM schemes at total throughputs of 4 and $6 \mathrm{~b} / \mathrm{s}$.

[33], our precoding-based SM regime jointly adapts the power 488 and the phases of the transmit signals and hence improves the 489 achievable BER performance.

Furthermore, in Fig. 6, we compare the QPSK-modulated 491 V-BLAST scheme and its PA-aided counterpart associated 492 with a zero-forcing-based successive interference cancelation 493 (ZF-SIC) detector [18] as the benchmarkers because their 494 detection complexity is similar to that of the single-stream 495 ML-based SM schemes. Observe in Fig. 6 for $m_{\text {all }}=4$ b/s that 496 our TPC-aided SM scheme outperforms the PA-aided VBLAST 497 arrangement relying on a ZF-SIC detector. Indeed, if a powerful 498 ML detector is employed for the VBLAST system, we can 499 achieve a better BER performance. However, designing PA 500 algorithms for ML-based VBLAST systems is a challenge, and 501 their detection complexity is high, as indicated in [34]. 502

Fig. 7 shows the frame error rate (FER) of both the proposed 503 precoded star-QAM-aided SM scheme and of the ASM scheme 504 [15]. The transmission frame size is $L_{F}=60 \mathrm{~b}^{3}{ }^{3}$ Note that, 505 although the proposed scheme and the ASM scheme exploit 506 the same degrees of freedom offered by the SM-MIMO for 507 improving the performance, our proposed scheme is capable 508 of avoiding the error propagation effects often experienced in 509 ASM, owing to ASM-mode signaling errors. Moreover, the 510 selection of TPC parameters is more flexible than that of ASM 511 because the modulation orders of ASM are selected from a 512 discrete set, whereas the TPC parameters are chosen from the 513 complex-valued field. As expected, the performance gain of 514 the proposed scheme over ASM is seen to be about $2 \mathrm{~dB}$ at 515 FER $=10^{-3}$ in Fig. 7.

\section{CONCLUSION}

In this paper, we have investigated the problem of designing 518 APM constellations that minimize the SM system's ABEP. 519 We considered a class of star-QAM constellations, which is 520

\footnotetext{
${ }^{3}$ Here, we assume that the channel matrix remains constant within each transmit frame and consider the FER performance of these schemes. Note that the ASM schemes often suffer from error-propagation effects, as indicated in Section I. Hence, using the FER comparison of the ASM and TPC-aided SM schemes may be more suitable than the BER metric.
} 
521 capable of flexibly adapting the ring ratios. We formulated 522 the constellation design problem as an optimization problem 523 and conceived an efficient iterative constellation-optimization 524 method. Moreover, a diagonal TPC technique was proposed 525 for the optimized star-QAM-aided SM to attain an improved 526 performance. The simulation results confirm that our proposed 527 optimized star-QAM-aided SM scheme outperforms the con528 ventional PSK/QAM schemes. Moreover, our TPC method also 529 exhibits an attractive BER/FER performance. For achieving an 530 improved performance for a high number of bits per symbol, 531 our further work will be focused on the integration of GSM and 532 channel coding into the proposed TPC schemes.

\section{REFERENCES}

1] R. Mesleh, H. Haas, S. Sinanovi, C. W. Ahn, and S. Yun, "Spatial modulation," IEEE Trans. Veh. Technol., vol. 57, no. 4, pp. 2228-2241, Jul. 2008.

[2] Y. Yang and B. Jiao, "Information-guided channel-hopping for high data rate wireless communication," IEEE Commun. Lett., vol. 12, no. 4, pp. 225-227, Apr. 2008.

3] J. Jeganathan, A. Ghrayeb, L. Szczecinski, and A. Ceron, "Space shift keying modulation for MIMO channels," IEEE Trans. Wireless Commun., vol. 8, no. 7, pp. 3692-3703, Jul. 2009.

4] M. Di Renzo, H. Haas, A. Ghrayeb, S. Sugiura, and L. Hanzo, "Spatial modulation for generalized MIMO: challenges, opportunities and implementation," Proc. IEEE, vol. 102, no. 1, pp. 56-103, Jan. 2014. [Online]. Available: http://eprints.soton.ac.uk/354175/

5] J. Wang, S. Jia, and J. Song, "Generalised spatial modulation system with multiple active transmit antennas and low complexity detection scheme," IEEE Trans. Wireless Commun., vol. 11, no. 4, pp. 1605-1615, Apr. 2012.

[6] E. Başar, Ü. Aygölü, E. Panayýrcý, and H. V. Poor, "Space-time block coded spatial modulation," IEEE Trans. Commun., vol. 59, no. 3, pp. 823832, Mar. 2011.

[7] P. Yang, Y. Xiao, Y. Yu, and S. Q. Li, "Adaptive spatial modulation for wireless MIMO transmission systems," IEEE Commun. Lett., vol. 15,

8] M. D. Renzo, H. Haas, and P. M. Grant, "Spatial modulation for multipleantenna wireless systems: A survey," IEEE Commun. Mag., vol. 49, no. 12 , pp. 182-191, Dec. 2011.

[9] L. Hanzo, S. X. Ng, T. Keller, and W. Webb, Quadrature Amplitude Modulation: From Basics to Adaptive Trellis-Coded, Turbo-Equalised and Space-Time Coded OFDM, CDMA and MC-CDMA Systems. Hoboken, NJ, USA: Wiley, 2004.

0] L. Hanzo, O. Alamri, M. El-Hajjar, and N. Wu, Near-Capacity MultiFunctional MIMO Systems: Sphere-Packing, Iterative Detection and Cooperation. Hoboken, NJ, USA: Wiley, 2009.

1] M. Di Renzo and H. Haas, "Bit error probability of spatial modulation (SM-) MIMO over generalized fading channels," IEEE Trans. Veh. Technol., vol. 61, no. 3, pp. 1124-1144, Mar. 2012.

2] P. Yang, Y. Xiao, L. Li, Q. Tang, Y. Yi, and S. Q. Li, "Link adaptation for spatial modulation with limited feedback," IEEE Trans. Veh. Technol., vol. 61 , no. 8, pp. 3808-3813, Oct. 2012.

3] J. Jeganathan, A. Ghrayeb, and L. Szczecinski, "Spatial modulation: optimal detection and performance analysis," IEEE Commun. Lett., vol. 12, no. 8, pp. 545-547, Aug. 2008.

4] S. Sugiura, C. Xu, S. X. Ng, and L. Hanzo, "Reduced-complexity coherent versus non-coherent QAM-aided space-time shift keying," IEEE Trans. Commun., vol. 59, no. 11, pp. 3090-3101, Nov. 2011.

5] P. Yang, Y. Xiao, Y. Yi, L. Li, Q. Tang, and S. Q. Li, "Simplified adaptive spatial modulation for limited-feedback MIMO," IEEE Trans. Veh. Technol., vol. 62, no. 6, pp. 2656-2666, Jul. 2013.

6] R. Y. Chang, S. J. Lin, and W. H. Chung, "Energy efficient transmission over space shift keying modulated MIMO channels," IEEE Tran. Commun., vol. 60, no. 12, pp. 2950-2959, Oct. 2012.

7] S. S. Ikki and R. Mesleh, "A general framework for performance analysis of space shift keying (SSK) modulation in the presence of Gaussian imperfect estimations," IEEE Commun. Lett., vol. 16, no. 2, pp. 228-230, Feb. 2012.

8] S. Sugiura and L. Hanzo, "On the joint optimization of dispersion matrices and constellations for near-capacity irregular precoded space-time shift keying," IEEE Trans. Wireless Commun., vol. 12, no. 1, pp. 380-387, Jan. 2013 no. 6, pp. 602-605, Jun. 2011.
[19] K. Ishibashi, W. Shin, H. Ochiai, and V. Tarokh, "A peak power efficient 592 cooperative diversity using star-QAM with coherent/noncoherent detec- 593 tion," IEEE Trans. Wireless Commun., vol. 12, no. 5, pp. 2137-2147, 594 May 2013.

[20] M. Di Renzo and H. Haas, "Improving the performance of space shift 596 keying (SSK) modulation via opportunistic power allocation," IEEE 597 Commun. Lett., vol. 14, no. 6, pp. 500-502, Jun. 2010.

[21] T. Handte, A. Muller, and J. Speidel, "BER analysis and optimization of 599 generalized spatial modulation in correlated fading channels," in Proc. 600 IEEE Veh. Technol. Conf. Fall, Sep. 2009, pp. 1-5.

[22] K. Ntontin, M. Di Renzo, A. Perez-Neira, and C. Verikoukis, "Adaptive 602 generalized space shift keying modulation," EURASIP J. Wireless Com- 603 mun. Netw., pp. 1-10, Feb. 2013.

[23] M. Maleki, H. Bahrami, S. Beygi, M. Kafashan, and N. H. Tran, "Space 605 modulation with CSI: constellation design and performance evalution," 606 IEEE Trans. Veh. Technol., vol. 62, no. 4, pp. 1623-1634, May 2013. 607

[24] B. M. Mthethwa and H. Xu, "Adaptive M-ary quadrature amplitude spatial 608 modulation," IET Commun., vol. 6, no. 18, pp. 3098-3108, Dec. 2012. 609

[25] L. L. Yang, "Transmitter preprocessing aided spatial modulation for 610 multiple-input multiple-output systems," in Proc. IEEE 73th Veh. Technol. 611 Conf.-Spring, Budapest, Hungary, May. 2011, pp. 15-18.

[26] A. Stavridis, S. Sinanovic, M. Di Renzo, and H. Haas, "Transmit precod- 613 ing for receive spatial modulation using imperfect channel knowledge," 614 in Proc. IEEE Veh. Technol. Conf. - Spring, Yokohama, Japan, May 6-9, 615 2012, pp. 1-5.

616

[27] L. Lampe, "Noncoherent coded modulation," Ph.D. dissertation, Dept. 617 Elect. Eng., Univ. Erlangen, Erlangen, Germany, 2002.

[28] X. Dong, N. C. Beaulieu, and P. H. Wittke, "Error probabilities of two- 619 dimensional $M$-ary signaling in fading," IEEE Trans. Commun., vol. 47, 620 no. 3, pp. 352-355, Mar. 1999.

[29] L. Szczecinski, H. Xu, X. Gao, and R. Bettancourt, "Efficient evaluation 622 of BER for arbitrary modulation and signaling in fading channels," IEEE 623 Trans. Commun., vol. 55, no. 11, pp. 2061-2064, Nov. 2007.

[30] A. Goldsmith, Wireless Communication. New York, NY, USA: 625 Cambridge Univ. Press, 2005, ch. 5.

[31] Y. T. Kim, H. Lee, S. Park, and I. Lee, "Optimal precoding for orthogo- 627 nalized spatial multiplexing in closed-loop MIMO systems," IEEE J. Sel. 628 Areas Commun., vol. 26, no. 8, pp. 1556-1567, Oct. 2008.

[32] S. Chen, S. Sugiura, and L. Hanzo, "Semi-blind joint channel estimation 630 and data detection for space-time shift keying systems," IEEE Signal 631 Process. Lett., vol. 17, no. 12, pp. 993-996, Dec. 2010.

[33] Y. Xiao, Q. Tang, L. Gong, P. Yang, and Z. Yang, Power scaling for spatial 633 modulation with limited feedback. [Online]. Available: http://downloads. 634 hindawi.com/journals/ijap/aip/718482.pdf

[34] S. H. Nam, O. S. Shin, and K. B. Lee, "Transmit power allocation for 636 a modified V-BLAST system," IEEE Trans. Commun., vol. 52, no. 7, 637 pp. 1074-1080, 2008.

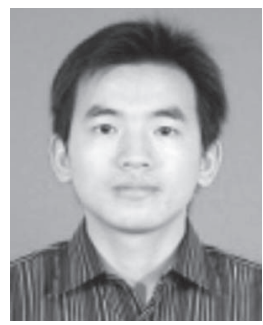

Ping Yang received the B.E. and M.E. degrees from 639 the University of Electronic Science and Technol- 640 ogy of China, Chengdu, China, in 2006 and 2009, 641 respectively, where he is currently working toward 642 the Ph.D. degree.

643

His research interests include multiple-input- 644 multiple-output systems, space-time coding, and 645 communication signal processing.

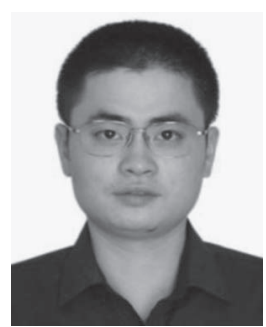

Yue Xiao received the Ph.D. degree in communi- 647 cation and information systems from the University 648 of Electronic Science and Technology of China, 649 Chengdu, China, in 2007.

$\mathrm{He}$ is currently an Associate Professor with the 651 University of Electronic Science and Technology of 652 China. He is the author of more than 30 international 653 journal articles and has been involved in several 654 projects in the Chinese Beyond 3G Communication 655 R\&D Program. His research interests include wire- 656 less and mobile communications. 


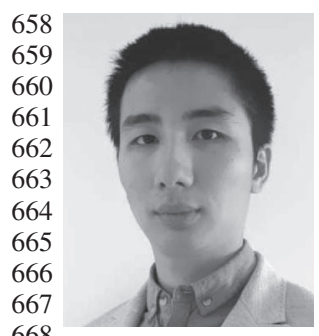

Bo Zhang received the B.S. degree in information engineering from the National University of Defense Technology, Changsha, China, in 2010. He is currently working toward the Ph.D. degree with the Communications, Signal Processing, and Control Group, School of Electronics and Computer Science, University of Southampton, Southampton, U.K.

His research interests include wireless communications, particularly the design and analysis of cooperative communications and network-coded networks.



Shaoqian Li (SM'12) received the B.Eng. degree in electrical engineering from the American University of Beirut, Beirut, Lebanon, in 2004 and the M.Sc. degree in radio-frequency communication systems and the Ph.D. degree in wireless communications from the University of Southampton, Southampton, U.K., in 2005 and 2008, respectively.

After his doctoral years, he joined Imagination Technologies as a Research Engineer, where he worked on designing and developing the bitinterleaved coded modulation peripherals in Imag680 ination's multistandard communications platform, which resulted in several 681 patent applications. Since January 2012, he has been a Lecturer with the 682 Communications, Signal Processing, and Control Group, School of Electronics 683 and Computer Science, University of Southampton. He is the author of a 684 Wiley-IEEE book and has written more than 40 journal and international 685 conference papers. His research interests include machine-to-machine com686 munications, millimeter-wave communications, large-scale multiple-input687 multiple-ouput systems, cooperative communications, and radio over fiber 688 systems.

689 Dr. Li has received several academic awards.

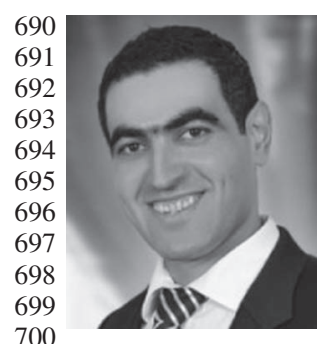

Mohammed El-Hajjar (M’08) received the B.Eng. degree in electrical engineering from the American University of Beirut, Beirut, Lebanon, in 2004 and the M.Sc. degree in radio-frequency communication systems and the Ph.D. degree in wireless communications from the University of Southampton, Southampton, U.K., in 2005 and 2008, respectively.

After his doctoral years, he joined Imagination Technologies as a Research Engineer, where he worked on designing and developing the bit701 interleaved coded modulation peripherals in Imagination's multistandard com702 munications platform, which resulted in several patent applications. Since 703 January 2012, he has been a Lecturer with the Communications, Signal Pro704 cessing, and Control Group, School of Electronics and Computer Science, Uni705 versity of Southampton. He is the author of a Wiley-IEEE book and has written 706 more than 40 journal and international conference papers. His main research 707 interests include the development of intelligent communications systems for 708 the Internet of Things, including energy-efficient transceiver design, cross-layer 709 optimization for large-scale networks, massive multiple-input-multiple-ouput 710 systems for millimeter-wave communications, cooperative communications, 711 and radio-over-fiber systems.

712 Dr. El-Hajjar has received several academic awards.

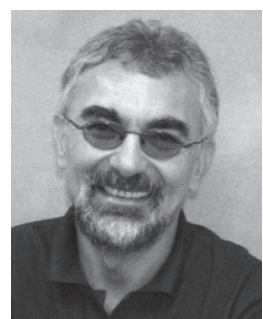

Lajos Hanzo (F'08) received the Master's degree 713 in electronics, the Ph.D. degree, and the Doctor 714 Honoris Causa degree from the Technical University 715 of Budapest, Budapest, Hungary, in 1976, 1983, and 716 2009, respectively.

During his 35-year career in telecommunications, 718 he has held various research and academic posts in 719 Hungary, Germany, and the U.K. From 2008 to 2012, 720 he was a Chaired Professor with Tsinghua Univer- 721 sity, Beijing, China. Since 1986, he has been with 722 the School of Electronics and Computer Science, 723 University of Southampton, Southampton, U.K., where he is currently the Chair 724 in telecommunications. He has successfully supervised $80 \mathrm{Ph}$.D. students. He 725 is the author or coauthor of 20 John Wiley/IEEE Press books on mobile radio 726 communications, totalling in excess of 10000 pages, and more than 1300727 research entries on IEEE Xplore. He has more than 16000 citations. He is 728 currently directing a 100-strong academic research team, working on a range of 729 research projects in the field of wireless multimedia communications sponsored 730 by industry, the Engineering and Physical Sciences Research Council, U.K., the 731 European Information Society Technology Programme, and the Mobile Virtual 732 Centre of Excellence, U.K. He is an enthusiastic supporter of industrial and 733 academic liaison, and he offers a range of industrial courses. His research is 734 funded by the European Research Council's Senior Research Fellow Grant. For 735 further information on research in progress and associated publications please 736 refer to http://www-mobile.ecs.soton.ac.uk.

Dr. Hanzo is a Fellow of the Royal Academy of Engineering, the Institution 738 of Engineering and Technology, and the European Association for Signal 739 Processing. He is also a Governor of the IEEE Vehicular Technology Society. 740 He has been a Technical Program Committee Chair and a General Chair of 741 IEEE conferences, has presented keynote lectures, and has been awarded a 742 number of distinctions. From 2008 to 2012, he was the Editor-in-Chief for the 743 IEEE Press. 


\section{AUTHOR QUERIES}

\section{AUTHOR PLEASE ANSWER ALL QUERIES}

AQ1 = There were discrepancies with the current affiliations of S. Li in the first footnote and that in the biography. Please check if the following changes are appropriate. If not, kindly provide the necessary corrections.

AQ2 = The sentence was modified for clarity. Please check if the following changes are appropriate. If not, kindly provide the necessary corrections.

AQ3 = Refs. [4] and [13] were the same and so was deleted from the list. Citations were renumbered accordingly. Please check.

\section{END OF ALL QUERIES}




\title{
Star-QAM Signaling Constellations for Spatial Modulation
}

\author{
Ping Yang, Yue Xiao, Bo Zhang, Shaoqian Li, Senior Member, IEEE, \\ Mohammed El-Hajjar, Member, IEEE, and Lajos Hanzo, Fellow, IEEE
}

\begin{abstract}
5 Abstract-The performance of spatial modulation (SM)-assisted 6 multiple-input-multiple-output (MIMO) communication systems 7 is highly dependent on the specific amplitude/phase modulation 8 (APM) signal constellation adopted. In this paper, we conceive 9 new star-quadrature amplitude modulation (star-QAM)-aided SM 10 schemes. Our goal is to minimize the system's average bit error 11 probability (ABEP). More specifically, a new class of star-QAM 12 constellations is introduced for $\mathbf{S M}$, which is capable of flexi13 bly adapting ring ratios of the amplitude levels. Then, under a 14 specific MIMO configuration and a predetermined transmission 15 rate, a simple and efficient ring-ratio optimization algorithm is 16 proposed to minimize the ABEP. Moreover, to improve further 17 the performance of our star-QAM-aided SM scheme, a diagonal 18 precoding technique is proposed, and a low-complexity minimum19 distance-based approach is conceived for extracting the precod20 ing parameters. Our numerical results show that the proposed 21 star-QAM-aided SM arrangement provides beneficial system per22 formance improvements compared with the identical-throughput 23 maximum-minimum distance (MMD) QAM and phase-shift key24 ing (PSK) benchmarkers. Moreover, our precoding scheme is 25 capable of further improving the attainable system performance 26 at a modest feedback requirement.
\end{abstract}

27 Index Terms-Constellation optimization, multiple-input28 multiple-output (MIMO), spatial modulation (SM), star29 quadrature amplitude modulation (star-QAM).

\section{INTRODUCTION}

31 PATIAL MODULATION (SM), which maps the informa32 tion bits to two information-carrying entities, namely the an33 tenna indexes and the combined amplitude/phase modulation 34 (APM) constellation, constitutes a promising low-complexity 35 multiple-input-multiple-output (MIMO) transmission tech36 nique [1]-[8]. In a conventional single-input-single-output 37 (SISO) system, the Gray-coded maximum-minimum distance

Manuscript received July 27, 2013; revised December 26, 2013; accepted February 8, 2014. This work was supported in part by the European Research Council under an Advanced Fellow Grant, by the National Science Foundation of China under Grant 61101101, by the Foundation Project of the National Key Laboratory of Science and Technology on Communications under Grant 9140C020404120C0201, and by the Key Laboratory of Universal Wireless Communications, Beijing University of Posts and Telecommunications, Chinese Ministry of Education, under Grant KFKT-2012102. The review of this paper was coordinated by Dr. G. Bauch.

P. Yang and Y. Xiao are with the National Key Laboratory of Science and Technology on Communications, University of Electronic Science and Technology of China, Chengdu 611731, China (e-mail: yplxw@163.com).

B. Zhang, S. Li, M. El-Hajjar, and L. Hanzo are with the School of Electronics and Computer Science, University of Southampton, Southampton SO17 1BJ, U.K. (e-mail: bz2g10@ecs.soton.ac.uk; 1sq@uestc.edu.cn; meh@ ecs.soton.ac.uk; 1h@ecs.soton.ac.uk).

Color versions of one or more of the figures in this paper are available online at http://ieeexplore.ieee.org.

Digital Object Identifier 10.1109/TVT.2014.2306986
(MMD) quadrature amplitude modulation (QAM) constellation 38 minimizes the bit error rate (BER) [9], [10]. However, the 39 advantage of MMD-QAM may be eroded in SM-MIMO sys- 40 tems [11]. This is due to the fact that the BER performance of 41 SM-MIMO systems is jointly determined by the spatial signal 42 (i.e., antenna indexes), by the classic APM constellation, and 43 by their interaction [11]-[18].

Recently, the effects of APM schemes on the performance 45 of SM have been investigated in [11], [14], and [18]. More 46 specifically, in [11], the performance of SM systems relying 47 both on conventional QAM and PSK modulation was studied, 48 demonstrating that, in some MIMO setups, the PSK-modulated 49 SM scheme may outperform the identical-throughput MMD- 50 QAM-aided SM scheme. In [18], the dispersion matrices and 51 the signal constellations were jointly optimized for a near- 52 capacity irregular precoded space-time shift keying (STSK) 53 system, which includes SM as a special case and strikes a 54 flexible rate-diversity tradeoff. It was also shown in [14] that 55 the star-QAM-aided STSK scheme outperforms its MMD- 56 based square-QAM-aided counterpart. This observation may 57 be also valid for SM systems [11]. The aforementioned results 58 indicated that the performance of SM is highly dependent on 59 the specific APM adopted; hence, a suitable APM scheme has 60 to be designed for this hybrid modulation scheme.

61

On the other hand, star-QAM constitutes a special case of 62 circular amplitude- and phase-shift keying, which is capable of 63 outperforming the classic square-QAM constellation in peak- 64 power-limited systems [19]. Hence, it has been adopted in most 65 of the recent satellite communication standards, such as in 66 the Digital Video Broadcast System (DVB) S2, DVB-SH, and 67 the Internet Protocol over Satellite and Advanced Broadcasting 68 System via Satellite [19]. The star-QAM constellation is com- 69 posed of multiple concentric circles, and it was shown to be 70 beneficial in the context of STSK systems. Hence, star-QAM 71 may be an attractive APM candidate for SM-MIMO. However, 72 the constellations' optimization has not been carried out for 73 star-QAM-aided SM.

Moreover, to increase the robustness of the SM-MIMO sys- 75 tem, limited-feedback-aided link adaptation schemes have been 76 proposed in [20]-[26]. For example, in [20], an opportunistic 77 power-allocation (PA) scheme was conceived for achieving a 78 beneficial transmit diversity gain in SM-MIMO systems. In 79 [21], a beamforming codebook was designed for optimizing the 80 coding gain of SM-MIMO based on the knowledge of the chan- 81 nel envelope's spatial correlation. Recently, an adaptive closed- 82 loop-aided method was invoked for providing both diversity 83 and coding gains in the context of space-shift keying (SSK)[22], 84 
85 which is a special case of SM. However, the scheme proposed 86 for SSK may not be directly applicable to the conventional 87 SM scheme. Moreover, ASM-MIMO architectures relying on 88 different combinations of modulation schemes were proposed 89 in [24], which aimed for maximizing the channel capacity at a 90 predefined target BER, rather than for minimizing the BER. In 91 contrast, in [25] and [26], a transmit precoding (TPC) technique 92 was used for improving the modulated signal design for SM. 93 However, this technique may only be suitable for a new class of 94 SM relying on a single-receiver antenna. For the conventional $95 \mathrm{SM}$, we proposed a near-instantaneously adaptive-modulation96 aided scheme for minimizing the BER [7], which was termed 97 adaptive SM (ASM). Then, we further generalized this paper in 98 [12] and [15], where the implementation complexity of ASM 99 was considerably reduced. However, ASM typically transmits 100 a different number of bits in the different-quality time slots, 101 which may be inconvenient in fixed-rate applications and po102 tentially leads to error propagation in the case of ASM-mode 103 signaling errors.

104 Against this background, the novel contributions of this 105 paper are threefold.

136 The remainder of this paper is organized as follows. In 137 Section II, we conceive a signaling constellation optimization 138 method for star-QAM-aided SM and elaborate both on the 139 choice of our optimization criterion and on the corresponding 140 optimization algorithm. In Section III, we propose a new TPC 141 scheme for enhancing the performance of the star-QAM-aided
SM. Our numerical analysis is carried out in Section IV. Finally, 142 our conclusions are presented in Section V.

\section{Signaling Constellation Optimization}

\section{A. Performance Metric and Star-QAM Constellation}

Consider a flat-fading MIMO channel associated with $N_{t} 146$ transmit antennas (TAs) and $N_{r}$ receive antennas. The $\left(N_{t} \times 147\right.$ 1)-element transmit symbol vector $\mathbf{x}$ is assumed to satisfy 148 $E\left[\mathbf{x x}^{H}\right]=\mathbf{I}_{N_{t}}$, where $\mathbf{I}_{N_{t}}$ denotes an $\left(N_{t} \times N_{t}\right)$-element 149 identity matrix. Then, the transmitted SM symbol $\mathbf{x} \in \mathbb{C}^{N_{t} \times 1} 150$ is given as $\mathbf{x}=s_{l}^{n} \mathbf{e}_{n}$ [21], where $s_{l}^{n}$ is the complex-valued 151 symbol of the APM scheme employed at the $n$th TA. For 152 example, $L$-PSK/QAM is associated with $m_{\mathrm{APM}}=\log _{2}(L) 153$ input bits, whereas $\mathbf{e}_{n}\left(1 \leq n \leq N_{t}\right)$ is selected from the $N_{t^{-}} 154$ dimensional standard basis vectors (i.e., $\mathbf{e}_{1}=[1,0, \ldots, 0]^{T}$ ), 155 according to $\log _{2}\left(N_{t}\right)$ input bits. The corresponding received 156 signal is given by

$$
\mathbf{y}=\mathbf{H x}+\mathbf{n}=\mathbf{h}_{n} s_{l}^{n}+\mathbf{n}
$$

where $\mathbf{H}$ is an $\left(N_{r} \times N_{t}\right)$-element channel matrix, $\mathbf{h}_{n}$ is the 158 $n$th column of $\mathbf{H}$, and the elements of the $N_{r}$-dimensional noise 159 vector $\mathbf{n}$ are Gaussian random variables obeying $\mathcal{C N}\left(0, N_{0}\right) . \quad 160$

In [11], an improved union bound partitions the ABEP 161 expression of SM-MIMO systems into three terms: the $P_{\text {spatial }} 162$ term related to the TA index, the $P_{\text {signal }}$ term related to the APM 163 signals, and the joint term $P_{\text {joint }}$, which depends on both the TA 164 index and on the APM signals. This bound is formulated as

$$
P_{\mathrm{SM}}(\rho) \leq P_{\text {spatial }}(\rho)+P_{\text {signal }}(\rho)+P_{\text {joint }}(\rho) .
$$

This improved union bound is more accurate than the 166 conventional union-bound-based methods, hence facilitating a 167 deeper understanding of the joint impact of spatial and APM 168 signals, as illustrated in [11]. We focus our attention on the sys- 169 tem's performance for transmission over i.i.d. Rayleigh fading 170 channels, which may be readily extended to the Nakagami-m 171 fading model of [11]. Let us assume that $\rho$ is the average SNR, 172 whereas $x_{l}$ and $x_{\hat{l}}$ represent two different APM constellation 173 points, with their modulus values being given as $\beta_{l}$ and $\beta_{\hat{l}}, 174$ respectively. Then, we have

$$
\begin{aligned}
& P_{\text {signal }}(\rho)=\frac{\log _{2}(L)}{\log _{2}\left(N_{t} \cdot L\right)} P_{\mathrm{APM}}(\rho) \\
& P_{\text {spatial }}(\rho)=\frac{\log _{2}\left(N_{t}\right) N_{t}}{2 L \log _{2}\left(N_{t} \cdot L\right)} \sum_{l=1}^{L} \mathcal{F}\left(\rho \beta_{l}^{2}\right) \\
& P_{\text {joint }}(\rho)=A \sum_{l=1}^{L} \sum_{\hat{l} \neq l=1}^{L}\left[B+C D_{H}\left(x_{l} \rightarrow x_{\hat{l}}\right)\right) \\
& \quad \times \mathcal{F}\left(\frac{\rho}{2}\left(\beta_{l}^{2}+\beta_{\hat{l}}^{2}\right)\right] .
\end{aligned}
$$

Here, $P_{\mathrm{APM}}(\rho)$ represents the error probability of conventional 176 $L$-APM, which depends on the ED of the constellation points 177 of APM, whereas $D_{H}\left(x_{l} \rightarrow x_{\hat{l}}\right)$ is the Hamming distance 178 between signals $x_{l}$ and $x_{\hat{l}}$. Here, $A=1 / L \log \left(N_{t} \cdot L\right), B=179$ $N_{t} \log \left(N_{t}\right) / 2$, and $C=\left(N_{t}-1\right)$ are constants for a fixed 180 


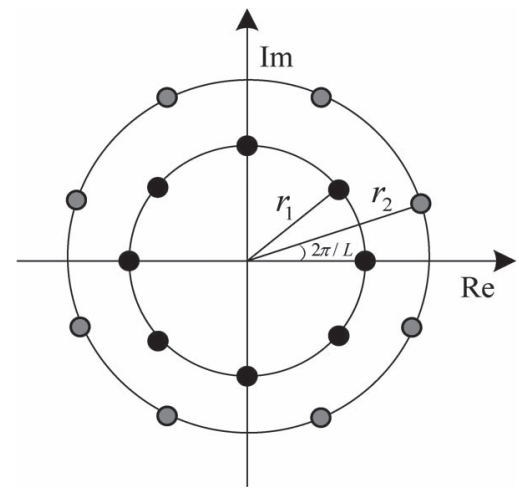

Fig. 1. Complex signal constellation of 16-star-QAM. The symbols are evenly distributed on two rings and the phase differences between the neighboring symbols on the same ring are equal.

181 MIMO setup. Moreover, the function $\mathcal{F}(\varepsilon)$ in (4) and (5) is the 182 pairwise error probability function [11], which is given by

$$
\mathcal{F}(\varepsilon)=\gamma(\varepsilon)^{N_{r}} \sum_{n=0}^{N_{r}-1}\left(\begin{array}{c}
N_{r}-1+n \\
n
\end{array}\right)[1-\gamma(\varepsilon)]^{n}
$$

183 where we have $\gamma(\varepsilon) \triangleq(1 / 2)(1-\sqrt{(\varepsilon / 2+\varepsilon)})$. Note that the 184 ABEP bound of (2) was proposed for the general family of 185 APM schemes, which contains not only the conventional PSK 186 but also the generic rectangular nonsquare-QAM schemes and 187 the square-QAM schemes. Moreover, since $P_{\text {signal }}$ is available 188 in closed form for conventional APM modulation schemes, the 189 bound of (2) is more accurate than the conventional results 190 of [21].

191 As indicated in (3)-(5), $P_{\text {signal }}$ mainly depends on the mini192 mum ED $d_{\min }$ of the APM constellation points, whereas $P_{\text {joint }}$ 193 and $P_{\text {spatial }}$ mainly depend on the modulus values $\beta_{l}(l=$ $1941, \ldots, L)$ of the APM constellation points.

195 Note that the modulus values $\beta_{l}$ are represented by the 196 Frobenius norms of the APM constellation points. These re197 sults suggested that, for jointly minimizing $P_{\text {signal }}, P_{\text {joint }}$, and $198 P_{\text {spatial }}$, we can focus our attention on the design of $d_{\min }$ and 199 on the $\beta_{l}$ parameters of APM.

200 To make the choice of the APM parameters $d_{\min }$ and $\beta_{l}$ as 201 flexible as possible, we consider a class of star-QAM constel202 lations, which subsumes the classic PSK as a special case but 203 may also be configured for maximizing the minimum ED of 204 the constellation by appropriately adjusting the ring ratios of 205 the amplitude levels. For simplicity, we consider the example 206 of a twin-ring 16-star-QAM constellation having a ring ratio 207 of $\alpha=r_{2} / r_{1}$, as shown in Fig. 1. The symbols are evenly 208 distributed on the two rings, and the phase differences between 209 the neighboring symbols on the same ring are equal. Unlike the 210 conventional twin-ring star-QAM constellation [19], [28], the 211 constellation points on the outer circle of our proposed star212 QAM constellation are rotated by $2 \pi / L$ degrees compared 213 with the corresponding constellation points on the inner circle 214 [27]. Hence, again, the conventional PSK constitutes an integral 215 part of our star-QAM scheme, which is associated with $\alpha=1$. 216 Table I summarizes the minimum EDs $d_{\min }$ between the con217 stellation points for different APM schemes. It is found that 218 this star-QAM scheme is capable of achieving almost the same
TABLE I

Minimum ED Between the Constellation POINTS FOR DIFFERENT APM SCHEMES

\begin{tabular}{|l|c|c|c|c|c|}
\hline Modulation order & 2 & 4 (MMD) & $8([9])$ & $16(\mathrm{MMD})$ & $32([9])$ \\
\hline PSK & 2 & $\sqrt{2}$ & 0.7654 & 0.3902 & 0.1960 \\
\hline QAM & -- & $\sqrt{2}$ & 0.8165 & 0.6325 & 0.4082 \\
\hline Proposed star-QAM & 2 & $\sqrt{2}$ & 0.9134 & 0.5737 & 0.3952 \\
\hline
\end{tabular}

minimum ED as the MMD-based QAM. Note that, although 219 this twin-ring star-QAM constellation has been indeed applied 220 for noncoherent detection [27], it has not been considered 221 whether this constellation can be directly applied to SM for 222 achieving performance improvements.

The aforementioned twin-ring philosophy of Fig. 1 may be 224 readily extended to multiple-ring star-QAM. The reasons for 225 considering twin-ring star-QAM in our paper are the following. 226

- It is an attractive APM modulation candidate for SM, 227 exhibiting a high performance at low detection complexity 228 compared with conventional QAM schemes, as detailed in 229 [13]-[15].

- It can be flexibly designed for different $d_{\min }$ and $\beta_{l}(l=231$ $1, \ldots, L)$ combinations, which is achieved by simply ad- 232 justing a single parameter $\alpha$, whereas $\beta_{l}$ can assume two 233 values because only two rings are considered.

- The ABEP of star-QAM, which is related to the $P_{\text {spatial }} 235$ term of (3), has been documented in [28] and [29].

\section{B. Optimization Criteria and Optimization Algorithm}

Observe in Fig. 1 that there are numerous options for the 238 parameter $\alpha$ of the star-QAM constellation, for a given MIMO 239 setup, specified by the total number of bits per symbol $m_{\text {all }}, 240$ the $\left(N_{r} \times N_{t}\right)$ configuration of transceiver, and the number of 241 modulation level $L$. The goal of star-QAM-aided signaling $242 \mathbf{A Q 2}$ constellation optimization is to find the specific ring ratio $\alpha, 243$ which minimizes the ABEP of the SM-MIMO of (2). Note that, 244 although the term $P_{\mathrm{SM}}(\rho)$ in (2) cannot be directly represented 245 by parameter $\alpha$, it varies as a function of $\alpha$, which may 246 be formulated as $P_{\mathrm{SM}}(\rho, \alpha)$. Following the aforementioned 247 approach, we formulated this optimization problem as

$$
\left\{\begin{array}{l}
\alpha^{*}=\min _{\alpha} P_{\mathrm{SM}}(\rho, \alpha) \\
\text { s.t. } \quad \alpha \geq 1
\end{array}\right.
$$

which may be a convex one for a fixed SNR value $\rho$, as 249 indicated in Fig. 4. However, deriving the closed-form solu- 250 tion of (7) remains an open challenge since the expression of 251 $P_{\mathrm{SM}}(\rho, \alpha)$ depends both on the specific APM constellation and 252 on the particular MIMO setup [19], and since the expressions 253 of $P_{\text {signal }}, P_{\text {joint }}$ and $P_{\text {spatial }}$ in (3)-(5) are complex. Hence, a 254 numerical search is adopted.

Our optimization algorithm conceived for finding the ring 256 ratio is summarized as follows.

Step 1: Initialize the values of $N_{r}, N_{t}, m_{\text {all }}, L$, and the SNR 258 value $\rho$. Set the iteration step size to $\Delta \alpha=0.1$ and the 259 number of iterations to $n=1$. The choice of $\Delta \alpha$ is flexible, 260 and a lower value of $\Delta \alpha$ may lead to a better performance. 261 We then set the search area of $\alpha$ to $1 \leq \alpha \leq U_{\alpha}$ and the 262 performance metric to $P_{\text {iter }}(n)=0$. 




Fig. 2. System model of the diagonal-precoding-assisted star-QAM-aided SM scheme.

264 Step 2: While $\alpha \leq U_{\alpha}$, let $\Delta \hat{\alpha}=\min \left\{\Delta \hat{\alpha}, U_{\alpha}-\alpha\right\}$, and cal265 culate the probabilities of $P_{\text {signal }}, P_{\text {joint }}$, and $P_{\text {spatial }}$ by us266 ing (3)-(5) associated with $\alpha$. Then, let $P_{\text {iter }}(n)=P_{\mathrm{SM}}(\rho)$ 267 using (2), and set $\alpha=\alpha+\Delta \hat{\alpha}$ and $n=n+1$.

268 Step 3 : Find the index $n^{*}=\min _{n}\left\{P_{\text {iter }}(n)\right\}$ to achieve the 269 optimal ring ratio of $\alpha^{*}=1+\left(n^{*}-1\right) \Delta \hat{\alpha}$.

270 In the aforementioned optimization algorithm, we have to 271 choose an appropriate $U_{\alpha}$ to promptly find the optimal $\alpha^{*}$. 272 More explicitly, an excessively low value of $U_{\alpha}$ may lead 273 to missing the optimal solution, whereas an excessively high 274 value of $U_{\alpha}$ imposes excessive computational complexity on 275 the optimization process. Hence, we will show in Section III 276 that $U_{\alpha}=3$ is a beneficial choice for promptly approaching 277 the optimal results. Moreover, the optimum ring ratio $\alpha^{*}$ is a 278 function of the SNR. However, we will show that the optimum 279 ratio approaches its asymptotic optimum as the SNR increases.

\section{III. Proposed Diagonal Precoding For \\ 281 Star-Quadrature Amplitude Modulation-Aided 282 SPATIAL MODUALTION}

283 Since the performance of the optimum maximum-likelihood 284 (ML) receiver depends on the FD of the received signal con285 stellation [30], we propose a new TPC based on maximizing 286 the FD for the family of star-QAM-aided SM-MIMO systems, 287 when limited channel state information is available at the 288 transmitter. Since the FD is increased by the TPC algorithm, 289 the proposed scheme is expected to provide a beneficial system 290 performance improvement. To retain all the single-RF-related 291 benefits of SM, we design the TPC matrix $\mathbf{P}$ to be diagonal. 292 The system model of the diagonal-TPC-assisted star-QAM293 aided SM scheme is shown in Fig. 2. To identify the specific 294 TPC parameters, which are capable of maximizing the FD, 295 we propose a low-complexity TPC design algorithm. We will 296 demonstrate that as few as two elements of the diagonal TPC 297 matrix have to be fed back to the transmitter, regardless of $N_{t}$.

\section{A. TPC Design Criterion}

299 To construct a TPC for star-QAM-aided SM-MIMO systems, 300 we can rewrite the system model of (1) as

$$
\mathbf{y}=\mathbf{H P x}+\mathbf{n}
$$

301 where $\mathbf{P}$ denotes the diagonal TPC matrix, which can be 302 represented as

$$
\mathbf{P}=\operatorname{diag}\left\{p_{1}, \ldots, p_{n}, \ldots, p_{N_{t}}\right\}
$$

where $p_{n}$ controls the channel gain associated with $x_{n}$. Here, 303 we let $\sum_{n=1}^{N_{t}}\left|p_{n}\right|^{2}=N_{t}$ for normalizing the transmit power. 304 Note that the introduction of TPC in SM does not affect the 305 advantages of SM, such as the avoidance of the interantenna 306 interference and the reliance on a single RF chain, because the 307 precoded transmit vector $\mathbf{P x}$ includes only a single nonzero 308 component; hence, only a single TA is activated in each time 309 slot, as indicated in (8).

310

Numerous techniques may be invoked for constructing the 311 TPC $\mathbf{P}$ [21], [25]. In this paper, similar to the precoding 312 methods conceived for the orthogonalized spatial multiplexing 313 of [31], we decompose $\mathbf{P}$ as

$$
\mathbf{P}=\overline{\mathbf{P}} \boldsymbol{\Theta}=\operatorname{diag}\left\{\bar{p}_{1} e^{j \theta_{1}}, \ldots, \bar{p}_{n} e^{j \theta_{n}}, \ldots, \bar{p}_{N_{t}} e^{j \theta_{N_{t}}}\right\}
$$

where $\overline{\mathbf{P}}=\operatorname{diag}\left\{\bar{p}_{1}, \ldots, \bar{p}_{n}, \ldots, \bar{p}_{N_{t}}\right\}$ represents the PA ma- 315 trix, whereas $\boldsymbol{\Theta}=\operatorname{diag}\left\{e^{j \theta_{1}}, \ldots, e^{j \theta_{n}}, \ldots, e^{j \theta_{N_{t}}}\right\}$ is the phase 316 rotation matrix. The FD between the constellation points at the 317 receiver is defined as

$$
\begin{aligned}
d_{\min }(\mathbf{H}, \mathbf{P}) & =\min _{\substack{\mathbf{x}_{i}, \mathbf{x}_{j} \in \mathbb{X}, \mathbf{x}_{i} \neq \mathbf{x}_{j}}}\left\|\mathbf{H P}\left(\mathbf{x}_{i}-\mathbf{x}_{j}\right)\right\|_{F} \\
& =\min _{\mathbf{e}_{i j} \in \mathbb{E}}\left\|\mathbf{H} \overline{\mathbf{P}} \boldsymbol{\Theta} \mathbf{e}_{i j}\right\|_{F}
\end{aligned}
$$

where $\mathbb{X}$ is the set of all legitimate transmit symbols, $\mathbf{e}_{i j}=319$ $\mathbf{x}_{i}-\mathbf{x}_{j}, i \neq j$ denotes the error vector, and $\mathbb{E}$ is a set of error 320 vectors. Then, we design the TPC $\mathbf{P}$ by maximizing the FD 321 with the aid of the following criterion:

$$
\left\{\begin{array}{l}
\mathbf{P}_{\mathrm{opt}}=\underset{\mathbf{P}}{\arg \max } d_{\min }(\mathbf{H}, \mathbf{P}) \\
\text { s.t. } \quad \sum_{n=1}^{N_{t}}\left|p_{n}\right|^{2}=N_{t} ; \quad p_{n} \in C ; \\
\\
\theta_{n} \in(0,2 \pi] ; \quad n=1, \ldots, N_{t} .
\end{array}\right.
$$

Note that, since the attainable performance of the optimum 323 single-stream ML receiver depends on the FD of the received 324 signal constellation [30], the maximization of the FD directly 325 reduces the probability of error. ${ }^{1}$ Let $\mathbf{x}_{i}=s_{l}^{i} \mathbf{e}_{i}$ and $\mathbf{x}_{j}=s_{k}^{j} \mathbf{e}_{j} 326$ denote two different transmit symbols, whereas $s_{l}^{i}$ and $s_{k}^{j} 327$ denote the constellation points $l$ and $k$ represented by the $i$ th 328 and $j$ th antennas, respectively. Then, the FD of (11) can be 329 represented as (13), where $\phi=\angle\left(\left(s_{l}^{i}\right)^{*} s_{k}^{j}\right)=-\left(s_{l}^{i}\left(s_{k}^{j}\right)^{*}\right)$. In 330

\footnotetext{
${ }^{1}$ Because the conventional PSK-and-QAM-aided SM scheme's performance is worse than that of the proposed star-QAM-aided SM, we only invoked the TPC algorithm for the star-QAM-aided SM for the sake of achieving further performance improvements. However, it is worth noting that the proposed TPC algorithm is also suitable for SM in conjunction with both conventional PSK and QAM schemes.
} 
331 the ASM scheme of [7], only the APM modulation orders to 332 be used by the transmitter are adapted, i.e., only the elements $333\left|s_{l}^{i}\right|,\left|s_{k}^{j}\right|$, and $\phi$ of (13), shown at the bottom of the page, are 334 dynamically adapted to the channel conditions, and the legit335 imate values of these elements are selected from the discrete 336 set depending on the modulation order set utilized. By contrast, 337 our proposed scheme adjusts all the TPC elements $\left|p_{i}\right|,\left|p_{j}\right|$, $338 \theta_{i}$, and $\theta_{j}$ of (13) for maximizing the FD $d_{\min }(\mathbf{H}, \mathbf{P})$, whose 339 legitimate values are drawn from the real-valued number field. 340 Based on these observations and on (13), the proposed scheme 341 and the ASM scheme may exploit the same degrees of freedom 342 as that offered by the SM-MIMO in terms of maximizing the 343 FD. However, unlike the ASM scheme of [7] and [15], our 344 proposed scheme assigns the same number of bits to each time 345 slot; hence, the potential error propagation effects experienced 346 in ASM are avoided.

\section{B. Low-Complexity TPC Design Algorithm}

348 To identify the specific TPC matrix $\mathbf{P}$, which is capable of 349 maximizing the $\mathrm{FD}$, we have to determine all the $N_{t}$ parameters $350 p_{n}\left(n=1, \ldots, N_{t}\right)$. Since it may become excessively complex 351 to jointly optimize these $N_{t}$ parameters in the complex-valued 352 field, we propose a low-complexity precoder design algorithm. 353 Similar to the one-bit reallocation algorithm designed for ASM 354 in [15], only the specific TA pair associated with the FD is con355 sidered, and the TPC parameters are selected for appropriately 356 weighting the SM symbols because the FD of this particular 357 TA pair predominantly determines the achievable performance. 358 The calculation of the TPC matrix is summarized in Fig. 3.

359 To be specific, given the channel matrix $\mathbf{H}$, the indexes of 360 the TA pair $(g, k)$ associated with the FD $d_{\min }(\mathbf{H})$ can be 361 found with the aid of the flowchart shown in Fig. 3. To offer 362 an increased FD, the precoding parameters of this TA pair can 363 be dynamically adapted. Note that, if the value of $g$ is the same 364 as $k$, it is plausible that the TA $g$ has the smallest channel gain. 365 In this case, the phase rotation elements of (10) do not have to 366 be considered because this would not increase the FD of (13). 367 To increase the FD, we only consider the PA matrix of (10) 368 and may deduct some power from the TA $u$ having the highest 369 channel gain, which may hence be reassigned it to the TA $g$. 370 As a result, $p_{u}$ and $p_{g}$ have to be optimized. On the other hand, 371 if the value of $g$ and $k$ is not the same, parameters $p_{g}$ and $p_{k}$ 372 have to be calculated. Overall, there are only two parameters, 373 namely, $p_{g}$ and $p_{k},\left(p_{u}\right.$ for $\left.g=k\right)$ that have to be searched 374 for. Finding the optimal values of $p_{g}$ and $p_{k}$ as a function of

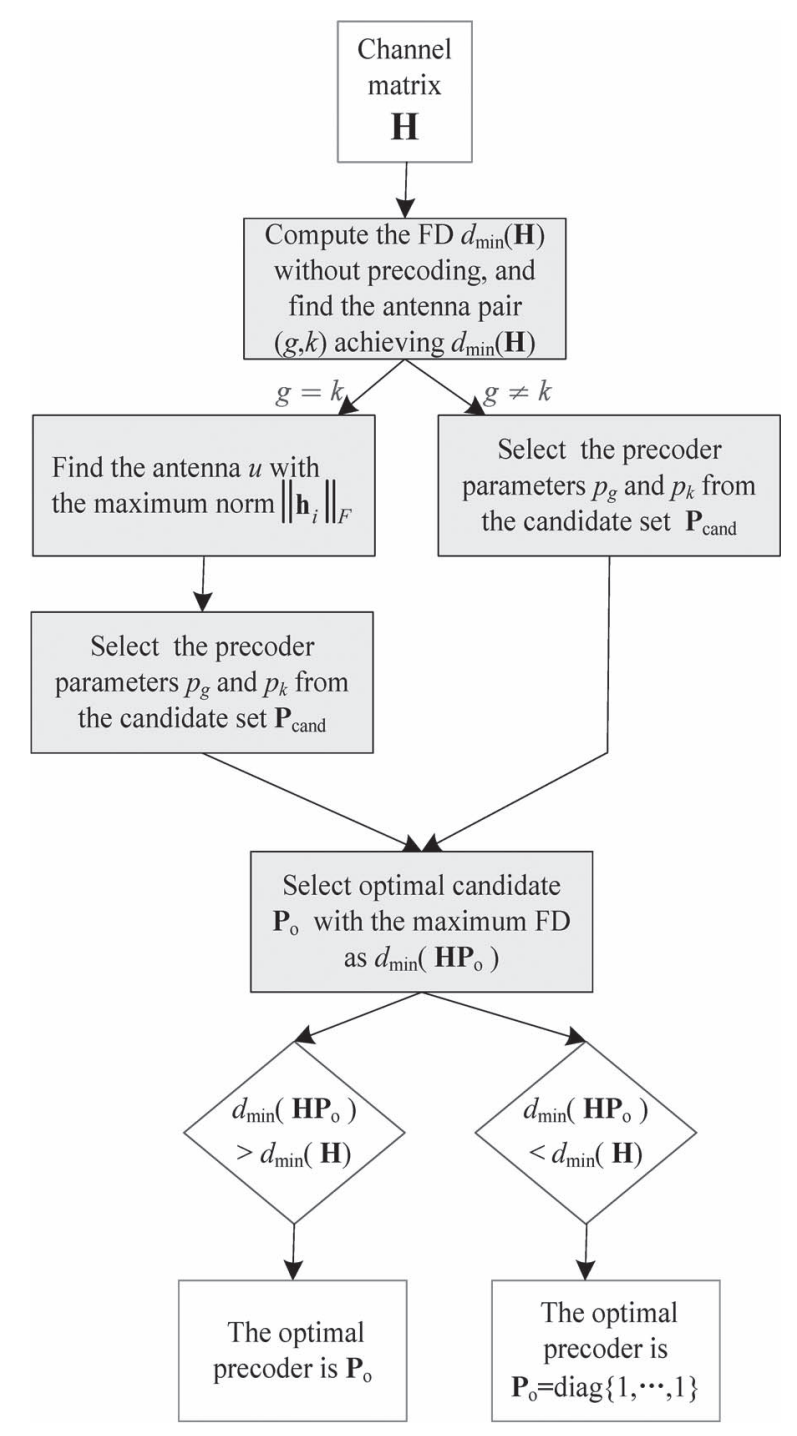

Fig. 3. Calculation of the diagonal precoding matrix for star-QAM-aided SM-MIMO.

both $\mathbf{H}$ and of the optimal transmit parameters involves an 375 exhaustive search over the vast design space of $\bar{p}_{g}, \bar{p}_{k}, \theta_{g}$, and 376 $\theta_{k}$ of (10), which is overly complex. To reduce the complexity, 377 according to (12), the power of the TA pair $(g, k)$ satisfies the 378 constraint $\bar{p}_{k}^{2}+\bar{p}_{g}^{2}=2$; hence, only the element $\bar{p}_{k}$ has to be 379 searched for in the power matrix $\overline{\mathbf{P}}$ of (10). Moreover, since 380 the phase rotation of the symbol is only carried by two TAs 381 and their phase difference is correlated, we can simplify the 382 computations by fixing $\theta_{k}=1$ and then finding the optimal 383

$$
\begin{aligned}
d_{\min }(\mathbf{H}, \mathbf{P}) & =\min _{s_{l}^{j}, s_{k}^{j} \in S}\left\|\mathbf{H P}\left(s_{l}^{i} \mathbf{e}_{i}-s_{k}^{j} \mathbf{e}_{j}\right)\right\|_{F} \\
& =\min _{s_{l}^{j}, s_{k}^{j} \in S}\left\|\left(\mathbf{h}_{i} p_{i} s_{l}^{i}-\mathbf{h}_{j} p_{j} s_{k}^{j}\right)\right\|_{F} \\
& =\min _{s_{l}^{j}, s_{k}^{j} \in S} \sqrt{\left|s_{l}^{i}\right|^{2}\left|p_{i}\right|^{2} \mathbf{h}_{i}^{\mathrm{H}} \mathbf{h}_{i}+\left|s_{k}^{j}\right|^{2}\left|p_{j}\right|^{2} \mathbf{h}_{j}^{\mathrm{H}} \mathbf{h}_{j}-2\left|p_{i}\right|\left|p_{j}\right|\left|s_{l}^{i}\right|\left|s_{k}^{j}\right| \operatorname{Re}\left\{\mathbf{h}_{i}^{\mathrm{H}} \mathbf{h}_{j} e^{j\left(\phi-\theta_{i}+\theta_{j}\right)}\right\}}
\end{aligned}
$$


$384 \theta_{g}$. This implies that only the phase parameter $\theta_{g}$ has to be 385 optimized for the phase matrix $\Theta$. In Fig. 3, a numerical search 386 is used for varying $\bar{p}_{g}$ and $\theta_{g}$ in small steps. Note that we 387 have $0 \leq \bar{p}_{g} \leq \sqrt{2}$ and $0 \leq \theta_{g} \leq 2 \pi$ according to (12). For our 388 numerical search, we have assumed

$$
\begin{cases}\bar{p}_{g}=\sqrt{2} / V_{1} * v_{1}, & v_{1}=0, \ldots, V_{1} \\ \theta_{g}=2 \pi / V_{2} * v_{2}, & v_{2}=0, \ldots, V_{2}\end{cases}
$$

389 where $V_{1}$ and $V_{2}$ represent the number of quantization steps and 390 can be flexibly selected according to the prevalent performance 391 requirements. As a result, the corresponding diagonal TPC 392 matrix candidates are

$$
\begin{gathered}
\mathbf{P}_{\text {cand }}=\operatorname{diag}\left\{1, \ldots, \bar{p}_{g} e^{j \theta_{g}}, \ldots, \sqrt{2-\bar{p}_{g}^{2}}, \ldots, 1\right\} \\
\uparrow g \text { th } \quad \uparrow k \text { th. }
\end{gathered}
$$

393 Upon denoting the quantized TPC matrix $\mathbf{P}$ as $\mathbf{P}_{\text {cand }}$, the 394 optimization problem of (12) is reformulated as

$$
\mathbf{P}_{\mathrm{opt}}=\underset{\left\{\mathbf{P} \in \mathbf{P}_{\text {cand }}, \mathbf{P}_{\mathrm{I}}\right\}}{\arg \max } d_{\min }(\mathbf{H}, \mathbf{P}) .
$$

395 where we have $\mathbf{P}_{\mathrm{I}}=\mathbf{I}_{N_{t}}$. In (16), the FD of the TPC matrices $396 \mathbf{P}_{\text {cand }}$ generated will be compared with that of the conventional 397 scheme associated with $\mathbf{P}_{\mathrm{I}}$, and then we select the one having 398 the largest FD as our final result. The receiver determines the 399 optimal diagonal TPC matrix based on (16) and feeds back the 400 TA indexes and their TPC parameters to the transmitter. Since 401 only the specific TA pair, which predominantly determines 402 the achievable performance, is considered, the proposed low403 complexity algorithm can be readily extended to a high number 404 of TAs.

\section{Simulation Results}

406 Here, we characterize the performance of both the proposed 407 star-QAM-aided SM scheme and of the corresponding TPC 408 scheme, and compare it with that of the conventional QAM409 modulated SM schemes, with the PSK-modulated SM schemes 410 and with the ASM schemes [15] for transmission over inde411 pendent Rayleigh block-flat MIMO channels. It is assumed that 412 the receiver is capable of perfect phase and gain tracking, i.e., 413 of perfect channel estimation. In practice, pilot symbols are 414 used for estimating the MIMO channel; hence, the estimated 415 channel matrix will inevitably be imperfect. To alleviate the 416 effects of channel estimation errors, the joint channel estimation 417 and data detection algorithm of [32] may be considered in the 418 proposed schemes, where the channel estimator and the data 419 detector iteratively exchange their information. We consider 420 two practical MIMO systems here, namely, $(2 \times 2)$ and $(4 \times$ $4214)$ MIMO systems. Moreover, in the TPC design algorithm, we 422 select $V_{1}=V_{2}=5$ for simplicity. ${ }^{2}$

423 Fig. 4 shows the optimal ring ratios of star-QAM-aided 424 SM relying on $(4 \times 4)$ elements for a different number of 425 modulation levels $L$, where the optimal ring ratio $\alpha^{*}$ is seen 426 to be a function of the SNR. The bound of (2) is well suited

\footnotetext{
${ }^{2}$ Note that the values of $V_{1}$ and $V_{2}$ can be different. Moreover, the selection of $V_{1}$ and $V_{2}$ is flexible, and higher values of $V_{1}$ and $V_{2}$ may lead to better performance at the cost of a higher TPC design complexity.
}

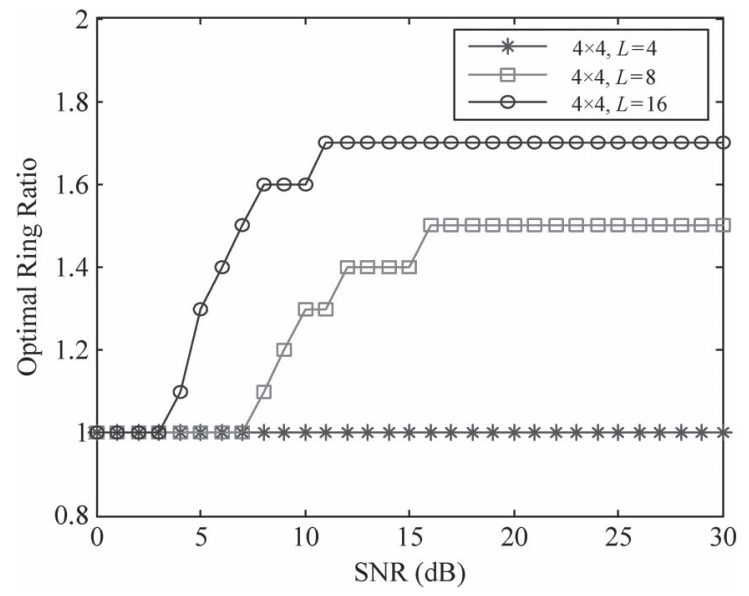

Fig. 4. Optimal ring ratios of star-QAM-aided SM with $(4 \times 4)$ MIMO for different numbers of modulation levels $L$.

for numerically optimizing the ring ratio, particularly in the 427 high-SNR region. Observe in Fig. 4 that the optimal ratios 428 approach their asymptotic values, as the SNR increases. This 429 is expected since the bound of (2) is also asymptotically tight 430 and the probability of an error event in slow fading associated 431 with ML detection is dominated by the minimum-distance error 432 event at high values of the SNR. Moreover, the optimal ring 433 ratios are different for different MIMO parameters.

434

Since the transmitter operates at a fixed ring ratio, we have 435 opted for the asymptotic ring-ratio value for the evaluation 436 of the BER. For example, we have chosen the optimal ring 437 ratio $\alpha^{*}=1.7$ for the 16 -star-QAM-aided $(4 \times 4)$ SM-MIMO, 438 according to the results in Fig. 4. This result may be readily 439 extended to other star-QAM-aided SM scenarios, such as the (4 440 $\times 4$ )-element star-QAM-aided SM schemes using $L=4,8$ in 441 Fig. 4.

In Figs. 5 and 6, we compare various SM-MIMO systems 443 relying on diverse MIMO parameters and modulation orders. 444 First, in Figs. 5 and 6, we depict the BER performance 445 of the conventional QAM-modulated SM schemes, of the 446 PSK-modulated SM arrangements, and of the proposed star- 447 QAM-aided SM scheme. Note that the optimized star-QAM 448 constellation is designed offline for different SM-MIMO sys- 449 tems. Hence, the resultant system does not need any feedback. 450 To be specific, we may create a parameter lookup table for 451 the star-QAM SM schemes associated with the MIMO setups 452 considered; hence, the complexity of the optimal ring-ratio 453 search process detailed in Section II is negligible. For com- 454 pleteness, we also included the theoretical upper bound [30] 455 for the family of conventional SM schemes. We found that 456 the conventional QAM-modulated SM scheme outperforms its 457 identical-throughput PSK counterpart for a $(4 \times 4)$-element 458 MIMO channel in Fig. 5, whereas the PSK scheme is preferred 459 for a $(2 \times 2)$-element MIMO channel in Fig. 6 . This indicates 460 that the best choice of the APM scheme depends on the specific 461 SM parameters, such as the MIMO setup and throughput. 462 Moreover, as shown in Fig. 5, the optimized star-QAM-aided 463 SM scheme provides an SNR gain of about $3 \mathrm{~dB}$ at BER $=464$ $10^{-5}$ over the conventional 16-PSK-modulated SM scheme and 465 an SNR gain of about $1.1 \mathrm{~dB}$ over the identical-throughput 466 


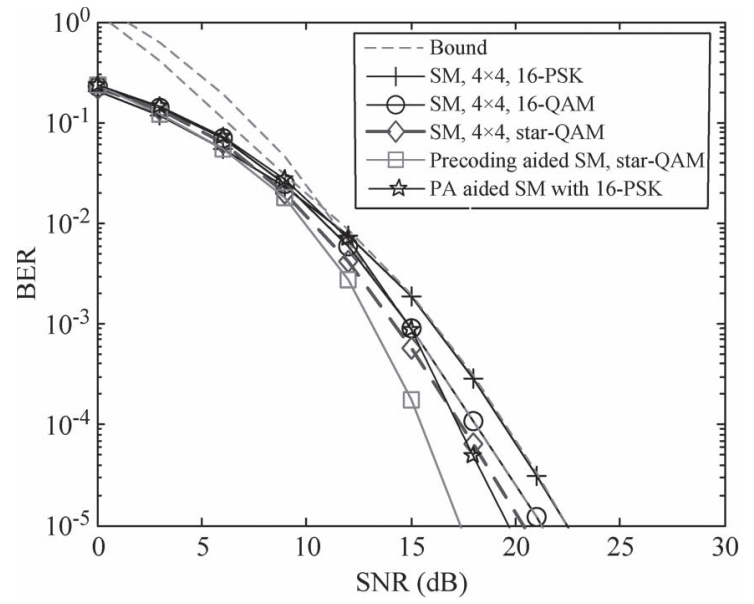

Fig. 5. BER performance of various SM-MIMO schemes operating in a $(4 \times$ 4) MIMO channel at a total throughput of $6 \mathrm{~b} / \mathrm{s}$. Since the transmitter operates with a fixed ring ratio, we have chosen the asymptotic ring-ratio value for the evaluation of star-QAM-aided schemes. Here, $\alpha$ is chosen as $\alpha=1.7$.

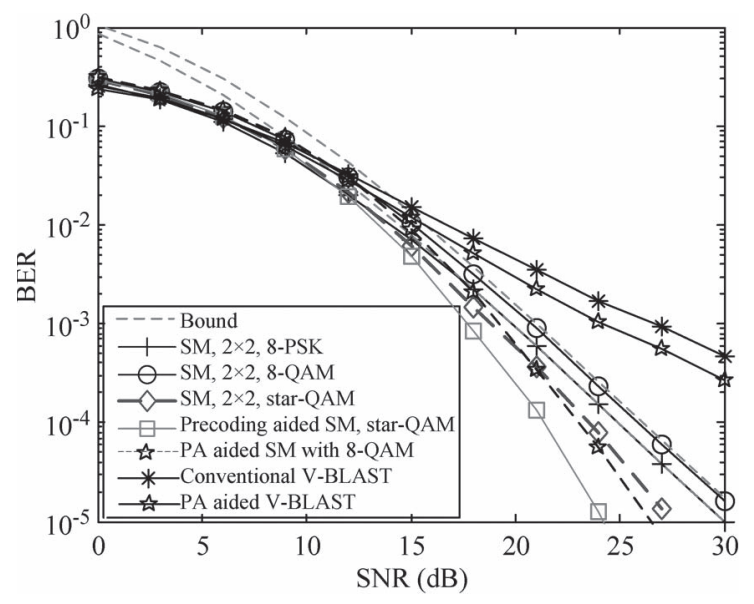

Fig. 6. BER performance of various SM schemes operating in $(2 \times 2)$ MIMO channel at a total throughput of $4 \mathrm{~b} / \mathrm{s}$. Here, $\alpha$ is chosen as $\alpha=1.5$.

467 Gray-coded MMD 16-QAM SM scheme. This advantage of the 468 optimized star-QAM scheme recorded for SM-MIMO is also 469 visible in Fig. 6.

470 Moreover, in Figs. 5 and 6, we also compare the 471 achievable BER performance of the limited-feedback-aided 472 ASM schemes. To be specific, two diagonal-precoding-aided 473 schemes, namely, the precoding-assisted star-QAM-based SM 474 schemes and the PA-aided SM schemes of [33] are compared. 475 For simplicity, the PA algorithm is only applied to the non476 ASM schemes exhibiting an inferior performance in Figs. 5 477 and 6, namely, to the conventional $(4 \times 4)$-element SM using 47816 -PSK and $(2 \times 2)$-element SM employing 8-QAM. Note that 479 the $(4 \times 4)$-element $\mathrm{SM}$ associated with 16 -QAM and $(2 \times$ 480 2)-element SM employing 8-PSK can also use the PA regime 481 to attain a BER improvement. Due to space limitations, these 482 results are not presented here. As shown in Figs. 5 and 6, the 483 proposed TPC schemes provide a gain of $2.5-3 \mathrm{~dB}$ at the BER 484 of $10^{-5}$ over the PA-aided SM schemes. This is because PA485 aided SM may be viewed as a special case of the proposed 486 precoding-aided SM created by only considering the PA matrix 487 in (10). To be specific, compared with the PA-aided SM of

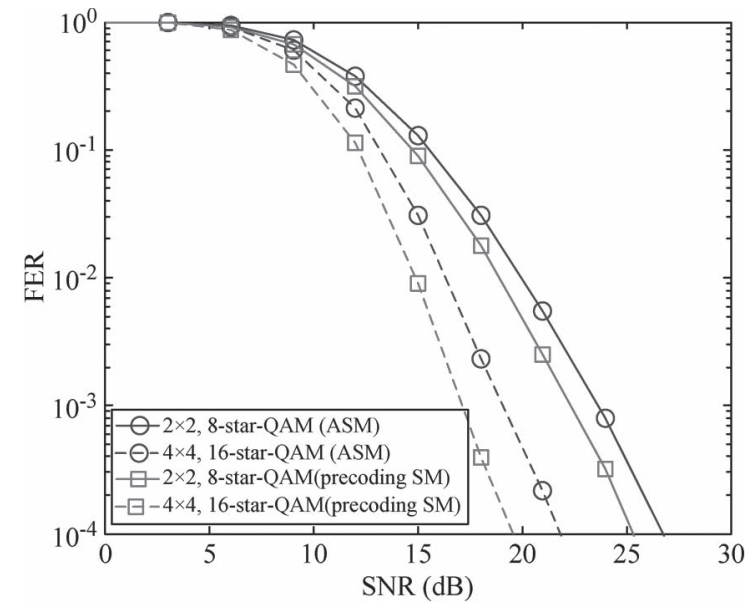

Fig. 7. FER performance of the proposed precoding star-QAM-aided SM and the ASM schemes at total throughputs of 4 and $6 \mathrm{~b} / \mathrm{s}$.

[33], our precoding-based SM regime jointly adapts the power 488 and the phases of the transmit signals and hence improves the 489 achievable BER performance.

490

Furthermore, in Fig. 6, we compare the QPSK-modulated 491 V-BLAST scheme and its PA-aided counterpart associated 492 with a zero-forcing-based successive interference cancelation 493 (ZF-SIC) detector [18] as the benchmarkers because their 494 detection complexity is similar to that of the single-stream 495 ML-based SM schemes. Observe in Fig. 6 for $m_{\text {all }}=4$ b/s that 496 our TPC-aided SM scheme outperforms the PA-aided VBLAST 497 arrangement relying on a ZF-SIC detector. Indeed, if a powerful 498 ML detector is employed for the VBLAST system, we can 499 achieve a better BER performance. However, designing PA 500 algorithms for ML-based VBLAST systems is a challenge, and 501 their detection complexity is high, as indicated in [34]. 502

Fig. 7 shows the frame error rate (FER) of both the proposed 503 precoded star-QAM-aided SM scheme and of the ASM scheme 504 [15]. The transmission frame size is $L_{F}=60 \mathrm{~b}^{3}{ }^{3}$ Note that, 505 although the proposed scheme and the ASM scheme exploit 506 the same degrees of freedom offered by the SM-MIMO for 507 improving the performance, our proposed scheme is capable 508 of avoiding the error propagation effects often experienced in 509 ASM, owing to ASM-mode signaling errors. Moreover, the 510 selection of TPC parameters is more flexible than that of ASM 511 because the modulation orders of ASM are selected from a 512 discrete set, whereas the TPC parameters are chosen from the 513 complex-valued field. As expected, the performance gain of 514 the proposed scheme over ASM is seen to be about $2 \mathrm{~dB}$ at 515 FER $=10^{-3}$ in Fig. 7.

\section{CONCLUSION}

In this paper, we have investigated the problem of designing 518 APM constellations that minimize the SM system's ABEP. 519 We considered a class of star-QAM constellations, which is 520

\footnotetext{
${ }^{3}$ Here, we assume that the channel matrix remains constant within each transmit frame and consider the FER performance of these schemes. Note that the ASM schemes often suffer from error-propagation effects, as indicated in Section I. Hence, using the FER comparison of the ASM and TPC-aided SM schemes may be more suitable than the BER metric.
} 
521 capable of flexibly adapting the ring ratios. We formulated 522 the constellation design problem as an optimization problem 523 and conceived an efficient iterative constellation-optimization 524 method. Moreover, a diagonal TPC technique was proposed 525 for the optimized star-QAM-aided SM to attain an improved 526 performance. The simulation results confirm that our proposed 527 optimized star-QAM-aided SM scheme outperforms the con528 ventional PSK/QAM schemes. Moreover, our TPC method also 529 exhibits an attractive BER/FER performance. For achieving an 530 improved performance for a high number of bits per symbol, 531 our further work will be focused on the integration of GSM and 532 channel coding into the proposed TPC schemes.

\section{REFERENCES}

1] R. Mesleh, H. Haas, S. Sinanovi, C. W. Ahn, and S. Yun, "Spatial modulation," IEEE Trans. Veh. Technol., vol. 57, no. 4, pp. 2228-2241, Jul. 2008.

[2] Y. Yang and B. Jiao, "Information-guided channel-hopping for high data rate wireless communication," IEEE Commun. Lett., vol. 12, no. 4, pp. 225-227, Apr. 2008.

3] J. Jeganathan, A. Ghrayeb, L. Szczecinski, and A. Ceron, "Space shift keying modulation for MIMO channels," IEEE Trans. Wireless Commun., vol. 8, no. 7, pp. 3692-3703, Jul. 2009.

4] M. Di Renzo, H. Haas, A. Ghrayeb, S. Sugiura, and L. Hanzo, "Spatial modulation for generalized MIMO: challenges, opportunities and implementation," Proc. IEEE, vol. 102, no. 1, pp. 56-103, Jan. 2014. [Online]. Available: http://eprints.soton.ac.uk/354175/

5] J. Wang, S. Jia, and J. Song, "Generalised spatial modulation system with multiple active transmit antennas and low complexity detection scheme," IEEE Trans. Wireless Commun., vol. 11, no. 4, pp. 1605-1615, Apr. 2012.

[6] E. Başar, Ü. Aygölü, E. Panayýrcý, and H. V. Poor, "Space-time block coded spatial modulation," IEEE Trans. Commun., vol. 59, no. 3, pp. 823832, Mar. 2011.

[7] P. Yang, Y. Xiao, Y. Yu, and S. Q. Li, "Adaptive spatial modulation for wireless MIMO transmission systems," IEEE Commun. Lett., vol. 15,

8] M. D. Renzo, H. Haas, and P. M. Grant, "Spatial modulation for multipleantenna wireless systems: A survey," IEEE Commun. Mag., vol. 49, no. 12 , pp. 182-191, Dec. 2011.

[9] L. Hanzo, S. X. Ng, T. Keller, and W. Webb, Quadrature Amplitude Modulation: From Basics to Adaptive Trellis-Coded, Turbo-Equalised and Space-Time Coded OFDM, CDMA and MC-CDMA Systems. Hoboken, NJ, USA: Wiley, 2004.

0] L. Hanzo, O. Alamri, M. El-Hajjar, and N. Wu, Near-Capacity MultiFunctional MIMO Systems: Sphere-Packing, Iterative Detection and Cooperation. Hoboken, NJ, USA: Wiley, 2009.

1] M. Di Renzo and H. Haas, "Bit error probability of spatial modulation (SM-) MIMO over generalized fading channels," IEEE Trans. Veh. Technol., vol. 61, no. 3, pp. 1124-1144, Mar. 2012.

2] P. Yang, Y. Xiao, L. Li, Q. Tang, Y. Yi, and S. Q. Li, "Link adaptation for spatial modulation with limited feedback," IEEE Trans. Veh. Technol., vol. 61 , no. 8, pp. 3808-3813, Oct. 2012.

3] J. Jeganathan, A. Ghrayeb, and L. Szczecinski, "Spatial modulation: optimal detection and performance analysis," IEEE Commun. Lett., vol. 12, no. 8, pp. 545-547, Aug. 2008.

4] S. Sugiura, C. Xu, S. X. Ng, and L. Hanzo, "Reduced-complexity coherent versus non-coherent QAM-aided space-time shift keying," IEEE Trans. Commun., vol. 59, no. 11, pp. 3090-3101, Nov. 2011.

5] P. Yang, Y. Xiao, Y. Yi, L. Li, Q. Tang, and S. Q. Li, "Simplified adaptive spatial modulation for limited-feedback MIMO," IEEE Trans. Veh. Technol., vol. 62, no. 6, pp. 2656-2666, Jul. 2013.

6] R. Y. Chang, S. J. Lin, and W. H. Chung, "Energy efficient transmission over space shift keying modulated MIMO channels," IEEE Tran. Commun., vol. 60, no. 12, pp. 2950-2959, Oct. 2012.

7] S. S. Ikki and R. Mesleh, "A general framework for performance analysis of space shift keying (SSK) modulation in the presence of Gaussian imperfect estimations," IEEE Commun. Lett., vol. 16, no. 2, pp. 228-230, Feb. 2012.

8] S. Sugiura and L. Hanzo, "On the joint optimization of dispersion matrices and constellations for near-capacity irregular precoded space-time shift keying," IEEE Trans. Wireless Commun., vol. 12, no. 1, pp. 380-387, Jan. 2013 no. 6, pp. 602-605, Jun. 2011.
[19] K. Ishibashi, W. Shin, H. Ochiai, and V. Tarokh, "A peak power efficient 592 cooperative diversity using star-QAM with coherent/noncoherent detec- 593 tion," IEEE Trans. Wireless Commun., vol. 12, no. 5, pp. 2137-2147, 594 May 2013.

[20] M. Di Renzo and H. Haas, "Improving the performance of space shift 596 keying (SSK) modulation via opportunistic power allocation," IEEE 597 Commun. Lett., vol. 14, no. 6, pp. 500-502, Jun. 2010.

[21] T. Handte, A. Muller, and J. Speidel, "BER analysis and optimization of 599 generalized spatial modulation in correlated fading channels," in Proc. 600 IEEE Veh. Technol. Conf. Fall, Sep. 2009, pp. 1-5.

[22] K. Ntontin, M. Di Renzo, A. Perez-Neira, and C. Verikoukis, "Adaptive 602 generalized space shift keying modulation," EURASIP J. Wireless Com- 603 mun. Netw., pp. 1-10, Feb. 2013.

[23] M. Maleki, H. Bahrami, S. Beygi, M. Kafashan, and N. H. Tran, "Space 605 modulation with CSI: constellation design and performance evalution," 606 IEEE Trans. Veh. Technol., vol. 62, no. 4, pp. 1623-1634, May 2013. 607

[24] B. M. Mthethwa and H. Xu, "Adaptive M-ary quadrature amplitude spatial 608 modulation," IET Commun., vol. 6, no. 18, pp. 3098-3108, Dec. 2012. 609

[25] L. L. Yang, "Transmitter preprocessing aided spatial modulation for 610 multiple-input multiple-output systems," in Proc. IEEE 73th Veh. Technol. 611 Conf.-Spring, Budapest, Hungary, May. 2011, pp. 15-18.

[26] A. Stavridis, S. Sinanovic, M. Di Renzo, and H. Haas, "Transmit precod- 613 ing for receive spatial modulation using imperfect channel knowledge," 614 in Proc. IEEE Veh. Technol. Conf. - Spring, Yokohama, Japan, May 6-9, 615 2012, pp. 1-5.

616

[27] L. Lampe, "Noncoherent coded modulation," Ph.D. dissertation, Dept. 617 Elect. Eng., Univ. Erlangen, Erlangen, Germany, 2002.

[28] X. Dong, N. C. Beaulieu, and P. H. Wittke, "Error probabilities of two- 619 dimensional $M$-ary signaling in fading," IEEE Trans. Commun., vol. 47, 620 no. 3, pp. 352-355, Mar. 1999.

[29] L. Szczecinski, H. Xu, X. Gao, and R. Bettancourt, "Efficient evaluation 622 of BER for arbitrary modulation and signaling in fading channels," IEEE 623 Trans. Commun., vol. 55, no. 11, pp. 2061-2064, Nov. 2007.

[30] A. Goldsmith, Wireless Communication. New York, NY, USA: 625 Cambridge Univ. Press, 2005, ch. 5.

[31] Y. T. Kim, H. Lee, S. Park, and I. Lee, "Optimal precoding for orthogo- 627 nalized spatial multiplexing in closed-loop MIMO systems," IEEE J. Sel. 628 Areas Commun., vol. 26, no. 8, pp. 1556-1567, Oct. 2008.

[32] S. Chen, S. Sugiura, and L. Hanzo, "Semi-blind joint channel estimation 630 and data detection for space-time shift keying systems," IEEE Signal 631 Process. Lett., vol. 17, no. 12, pp. 993-996, Dec. 2010.

[33] Y. Xiao, Q. Tang, L. Gong, P. Yang, and Z. Yang, Power scaling for spatial 633 modulation with limited feedback. [Online]. Available: http://downloads. 634 hindawi.com/journals/ijap/aip/718482.pdf

[34] S. H. Nam, O. S. Shin, and K. B. Lee, "Transmit power allocation for 636 a modified V-BLAST system," IEEE Trans. Commun., vol. 52, no. 7, 637 pp. 1074-1080, 2008.

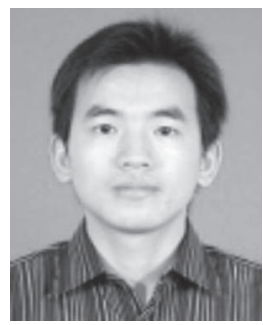

Ping Yang received the B.E. and M.E. degrees from 639 the University of Electronic Science and Technol- 640 ogy of China, Chengdu, China, in 2006 and 2009, 641 respectively, where he is currently working toward 642 the Ph.D. degree.

643

His research interests include multiple-input- 644 multiple-output systems, space-time coding, and 645 communication signal processing.

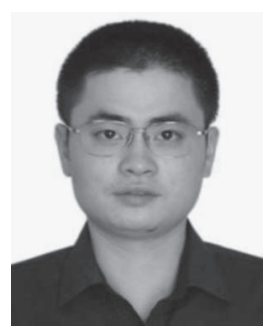

Yue Xiao received the Ph.D. degree in communi- 647 cation and information systems from the University 648 of Electronic Science and Technology of China, 649 Chengdu, China, in 2007.

$\mathrm{He}$ is currently an Associate Professor with the 651 University of Electronic Science and Technology of 652 China. He is the author of more than 30 international 653 journal articles and has been involved in several 654 projects in the Chinese Beyond 3G Communication 655 R\&D Program. His research interests include wire- 656 less and mobile communications. 


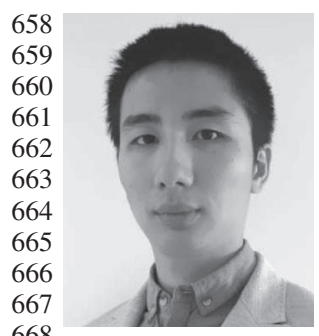

Bo Zhang received the B.S. degree in information engineering from the National University of Defense Technology, Changsha, China, in 2010. He is currently working toward the Ph.D. degree with the Communications, Signal Processing, and Control Group, School of Electronics and Computer Science, University of Southampton, Southampton, U.K.

His research interests include wireless communications, particularly the design and analysis of cooperative communications and network-coded networks.

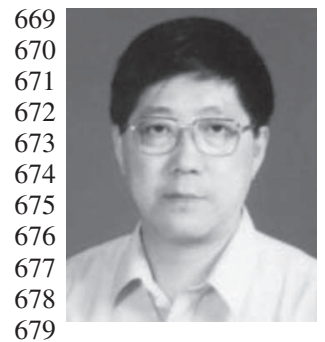

Shaoqian Li (SM'12) received the B.Eng. degree in electrical engineering from the American University of Beirut, Beirut, Lebanon, in 2004 and the M.Sc. degree in radio-frequency communication systems and the Ph.D. degree in wireless communications from the University of Southampton, Southampton, U.K., in 2005 and 2008, respectively.

After his doctoral years, he joined Imagination Technologies as a Research Engineer, where he worked on designing and developing the bitinterleaved coded modulation peripherals in Imag680 ination's multistandard communications platform, which resulted in several 681 patent applications. Since January 2012, he has been a Lecturer with the 682 Communications, Signal Processing, and Control Group, School of Electronics 683 and Computer Science, University of Southampton. He is the author of a 684 Wiley-IEEE book and has written more than 40 journal and international 685 conference papers. His research interests include machine-to-machine com686 munications, millimeter-wave communications, large-scale multiple-input687 multiple-ouput systems, cooperative communications, and radio over fiber 688 systems.

689 Dr. Li has received several academic awards.

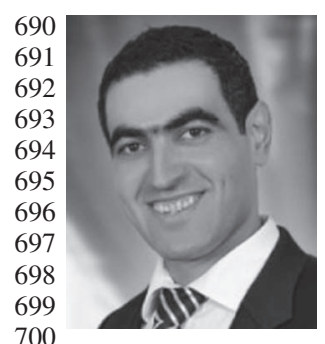

Mohammed El-Hajjar (M’08) received the B.Eng. degree in electrical engineering from the American University of Beirut, Beirut, Lebanon, in 2004 and the M.Sc. degree in radio-frequency communication systems and the Ph.D. degree in wireless communications from the University of Southampton, Southampton, U.K., in 2005 and 2008, respectively.

After his doctoral years, he joined Imagination Technologies as a Research Engineer, where he worked on designing and developing the bit701 interleaved coded modulation peripherals in Imagination's multistandard com702 munications platform, which resulted in several patent applications. Since 703 January 2012, he has been a Lecturer with the Communications, Signal Pro704 cessing, and Control Group, School of Electronics and Computer Science, Uni705 versity of Southampton. He is the author of a Wiley-IEEE book and has written 706 more than 40 journal and international conference papers. His main research 707 interests include the development of intelligent communications systems for 708 the Internet of Things, including energy-efficient transceiver design, cross-layer 709 optimization for large-scale networks, massive multiple-input-multiple-ouput 710 systems for millimeter-wave communications, cooperative communications, 711 and radio-over-fiber systems.

712 Dr. El-Hajjar has received several academic awards.

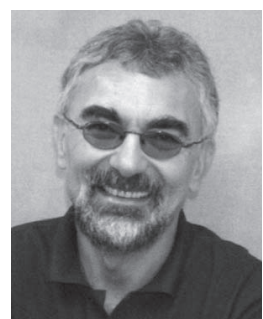

Lajos Hanzo (F'08) received the Master's degree 713 in electronics, the Ph.D. degree, and the Doctor 714 Honoris Causa degree from the Technical University 715 of Budapest, Budapest, Hungary, in 1976, 1983, and 716 2009, respectively.

During his 35-year career in telecommunications, 718 he has held various research and academic posts in 719 Hungary, Germany, and the U.K. From 2008 to 2012, 720 he was a Chaired Professor with Tsinghua Univer- 721 sity, Beijing, China. Since 1986, he has been with 722 the School of Electronics and Computer Science, 723 University of Southampton, Southampton, U.K., where he is currently the Chair 724 in telecommunications. He has successfully supervised $80 \mathrm{Ph}$.D. students. He 725 is the author or coauthor of 20 John Wiley/IEEE Press books on mobile radio 726 communications, totalling in excess of 10000 pages, and more than 1300727 research entries on IEEE Xplore. He has more than 16000 citations. He is 728 currently directing a 100-strong academic research team, working on a range of 729 research projects in the field of wireless multimedia communications sponsored 730 by industry, the Engineering and Physical Sciences Research Council, U.K., the 731 European Information Society Technology Programme, and the Mobile Virtual 732 Centre of Excellence, U.K. He is an enthusiastic supporter of industrial and 733 academic liaison, and he offers a range of industrial courses. His research is 734 funded by the European Research Council's Senior Research Fellow Grant. For 735 further information on research in progress and associated publications please 736 refer to http://www-mobile.ecs.soton.ac.uk.

Dr. Hanzo is a Fellow of the Royal Academy of Engineering, the Institution 738 of Engineering and Technology, and the European Association for Signal 739 Processing. He is also a Governor of the IEEE Vehicular Technology Society. 740 He has been a Technical Program Committee Chair and a General Chair of 741 IEEE conferences, has presented keynote lectures, and has been awarded a 742 number of distinctions. From 2008 to 2012, he was the Editor-in-Chief for the 743 IEEE Press. 


\section{AUTHOR QUERIES}

\section{AUTHOR PLEASE ANSWER ALL QUERIES}

AQ1 = There were discrepancies with the current affiliations of S. Li in the first footnote and that in the biography. Please check if the following changes are appropriate. If not, kindly provide the necessary corrections.

AQ2 = The sentence was modified for clarity. Please check if the following changes are appropriate. If not, kindly provide the necessary corrections.

AQ3 = Refs. [4] and [13] were the same and so was deleted from the list. Citations were renumbered accordingly. Please check.

\section{END OF ALL QUERIES}

